\title{
Update on geological and geophysical investigations in the Slate Creek mining area, Alaska
}

\author{
Jennifer E. Athey ${ }^{1}$, Lawrence K. Freeman ${ }^{1}$, Chad P. Hults ${ }^{2}$, David J. Szumigala ${ }^{1}$, \\ Melanie B. Werdon ${ }^{1}$, Richard D. Koehler ${ }^{1}$, and Laurel E. Burns ${ }^{1}$ \\ ${ }^{1}$ Alaska Division of Geological \& Geophysical Surveys (DGGS) \\ ${ }^{2}$ U.S. Geological Survey, Alaska Science Center (USGS)
}

Presented by Jennifer Athey at the Alaska Miners Association meeting in Anchorage, Alaska on November 5, 2009.

In July 2009, the Alaska Division of Geological \& Geophysical Surveys (DGGS) conducted geologic mapping in the Slate Creek area 20 miles northeast of Paxson and immediately south of the Denali fault (parts of Mount Hayes A-2 and A-3 quadrangles). As part of the Airborne Geophysical/Geological Mineral Inventory (AGGMI) Program, we are utilizing new detailed airborne electromagnetic and magnetic geophysical data for the Slate Creek-Slana River area (Burns et al., 2009), whole rock data, ${ }^{40} \mathrm{Ar} /{ }^{39} \mathrm{Ar}$ and detrital zircon ages, thin-section and grain-mount petrography, palynology and macrofossils, historical, and industry data in conjunction with field observations to produce a 1:50,000-scale geologic map. The area has high mineral interest and is a key location for understanding the active Denali fault system. DGGS plans to publish the map on our website (http://www.dggs.alaska.gov/) in winter 2010.

The Slate Creek fault, a high-angle fault system parallel and possibly related to the Denali fault system, separates two dissimilar sections of bedrock described by Nokleberg et al. (1992). South of the Slate Creek fault, volcaniclastic rocks, thin limestones, volcanic sedimentary rocks, and basaltic to rhyolitic (Athey, 1999) "coherent" volcanic rocks compose the Permian-Pennsylvanian Slana Spur Formation. Red, oxidized quartzpyrite gossans spatially associated with quartz-phenocryst-bearing volcanic rocks contain up to 0.72 ounces of gold/ton (Athey, 1999). Farther south, near the edge of the study area, five or six 0.5- to 2-mile-wide, granite to basalt bodies of unknown age and chemistry intrude volcanic rocks of the Slana Spur Formation.

North of the Slate Creek fault, Early Permian Eagle Creek limestone and sedimentary rocks, Late Triassic Nikolai greenstone and minor sediments, Late Triassic Chitistone limestone, and Cretaceous to Jurassic argillite and phyllite compose the upper plate of an apparent north-dipping thrust fault. The lower plate of the thrust fault includes the felsic Slana Spur(?) and Eagle Creek formations, and Tertiary sedimentary rocks. The involvement of Tertiary sedimentary rocks in the thrust fault and the Slate Creek fault system infers a Tertiary, possibly Neogene, age of latest movement on the faults. Two hand-dug trenches on the Denali fault revealed evidence of possible Holocene offsets at this location in addition to the 2002 event. No Quaternary fault movement, other than on the Denali fault, was observed in air photos or outcrop in the study area.

Most of the 183,356 troy ounces of gold (Szumigala et al., 2009) and minor platinum group elements (PGE) recorded within the Chistochina mining district were extracted from placers in the Slate Creek area. Placer miners and previous researchers assert that placer gold in Quaternary valley and bench deposits was derived and reconcentrated from 
semi-consolidated to unconsolidated "round wash" gravels found on higher slopes and ridgetops between Slate Creek and the Chistochina Glacier. The "round wash" gravels, containing up to $113 \mathrm{ppm}$ gold (Bittenbender et al., 2007), eroded from fault-bounded and perched, poorly indurated Tertiary conglomerate. In addition to detailed mapping, we are conducting petrologic and palynological studies of the conglomerate to better understand the tectonic history of the area. PGEs in the placer deposits are likely sourced from one or more of the Triassic and (or) Cretaceous(?) mafic and ultramafic bodies in the study area. Planned chemical analyses and age data will help us test their PGE potential, and determine the structural (and intrusive?) relationships of these bodies relative to surrounding units.

Athey, J.E., 1999, Characterization of the DAT Zone, eastern Alaska Range, Alaska - A Calcic $\mathrm{Fe}-\mathrm{Cu}-\mathrm{Au}$ skarn prospect: Fairbanks, Alaska, University of Alaska, Master of Science thesis, $152 \mathrm{p}$.

Bittenbender, P.E., Bean, K.W., Kurtak, J.M., and Deininger, J.W., Jr., 2007, Mineral assessment of the Delta River mining district area, east-central Alaska: U.S. Bureau of Land Management Alaska Technical Report 57, 676 p., 2 sheets.

Burns, L.E., Fugro Airborne Surveys Corp., and Stevens Exploration Management Corp., 2009, Line, grid, and vector data, and maps for the airborne geophysical survey of the Slate Creek-Slana River Survey, Chistochina mining district, south-central Alaska: Alaska Division of Geological \& Geophysical Surveys Geophysical Report 2009-1, 18 sheets, 1 DVD, scale 1:63,360.

Nokleberg, W.J., Aleinikoff, J.N., Lange, I.M., Silva, S.R., Miyaoka, R.T., Schwab, C.E., and Zehner, R.E., 1992, Preliminary geologic map of the Mount Hayes quadrangle, eastern Alaska Range, Alaska: U.S. Geological Survey Open-File Report 92-594, 39 p., 1 sheet, scale 1:250,000.

Szumigala, D.J., Hughes, R.A., and Harbo, L.A., 2009, Alaska's Mineral Industry 2008: Alaska Division of Geological \& Geophysical Surveys Special Report 63, 89 p. 


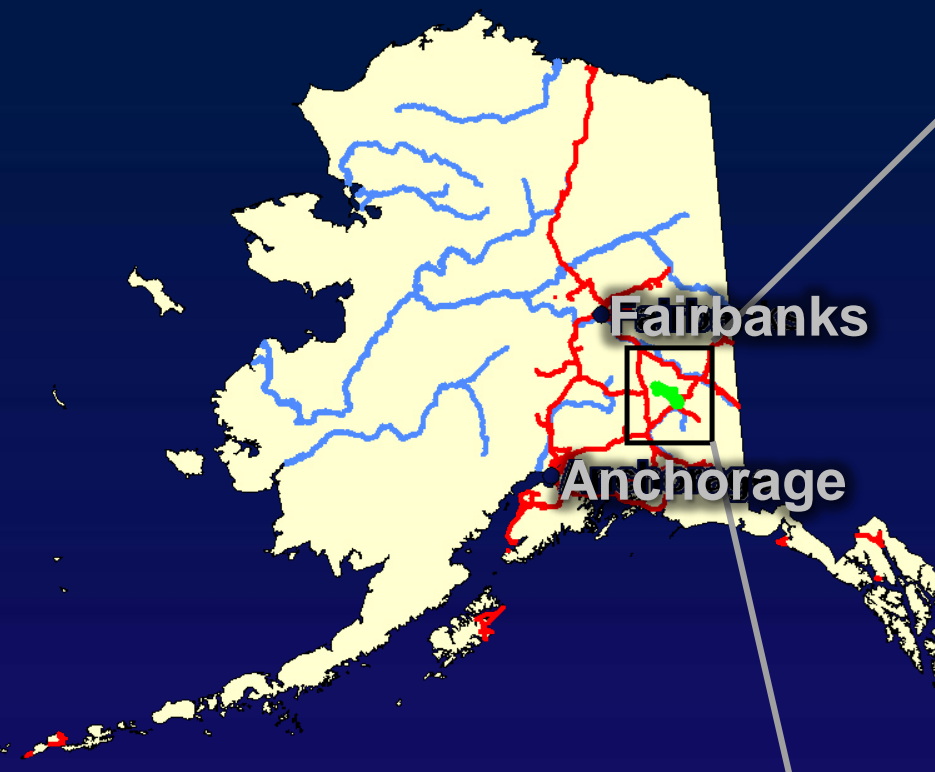

Slate Creek area, Chistochina mining district

183,356 Troy ounces Au, Minor amounts of PGE's

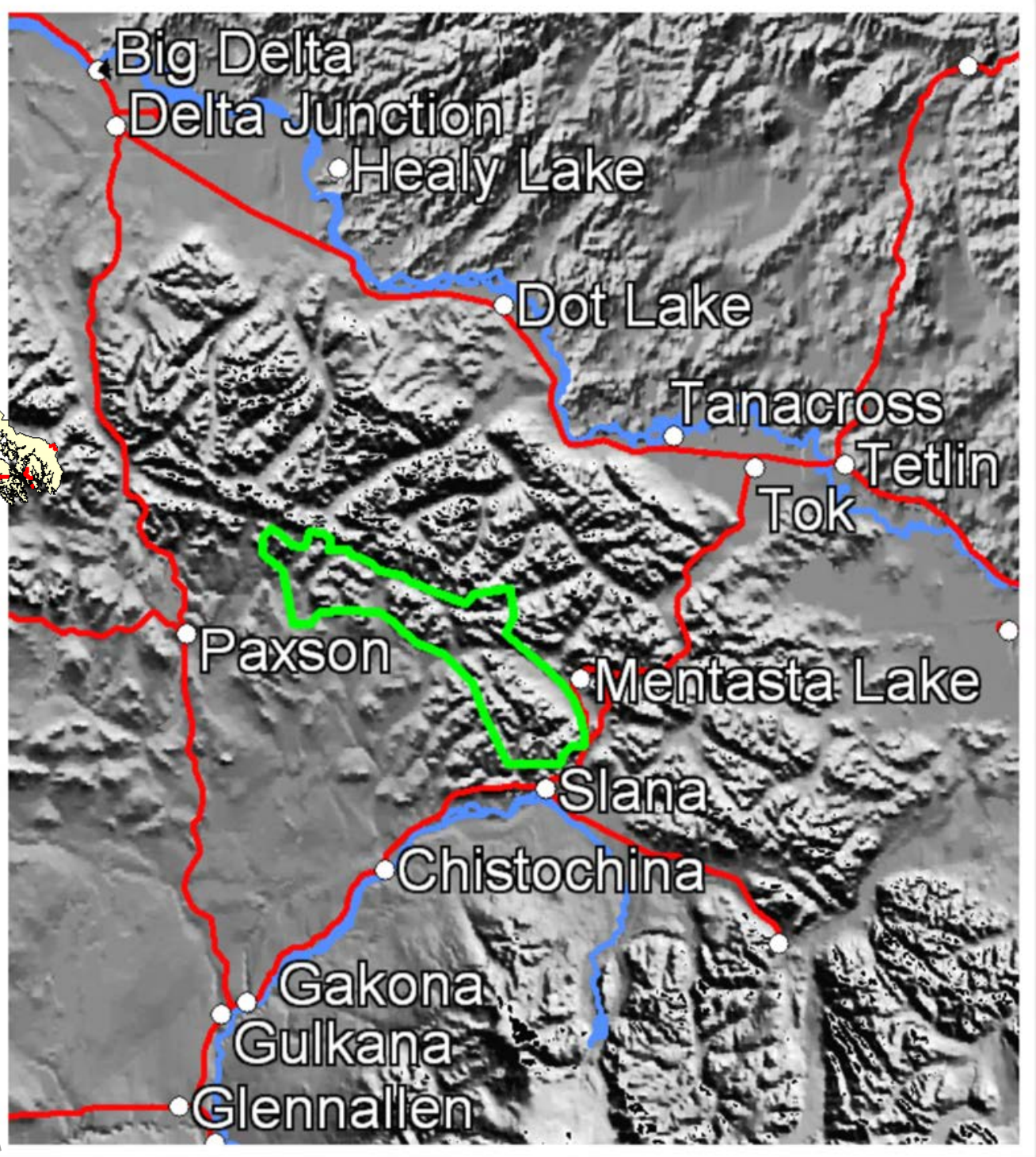

50 miles 


\section{ARDF Placer and Lode Occurrences}

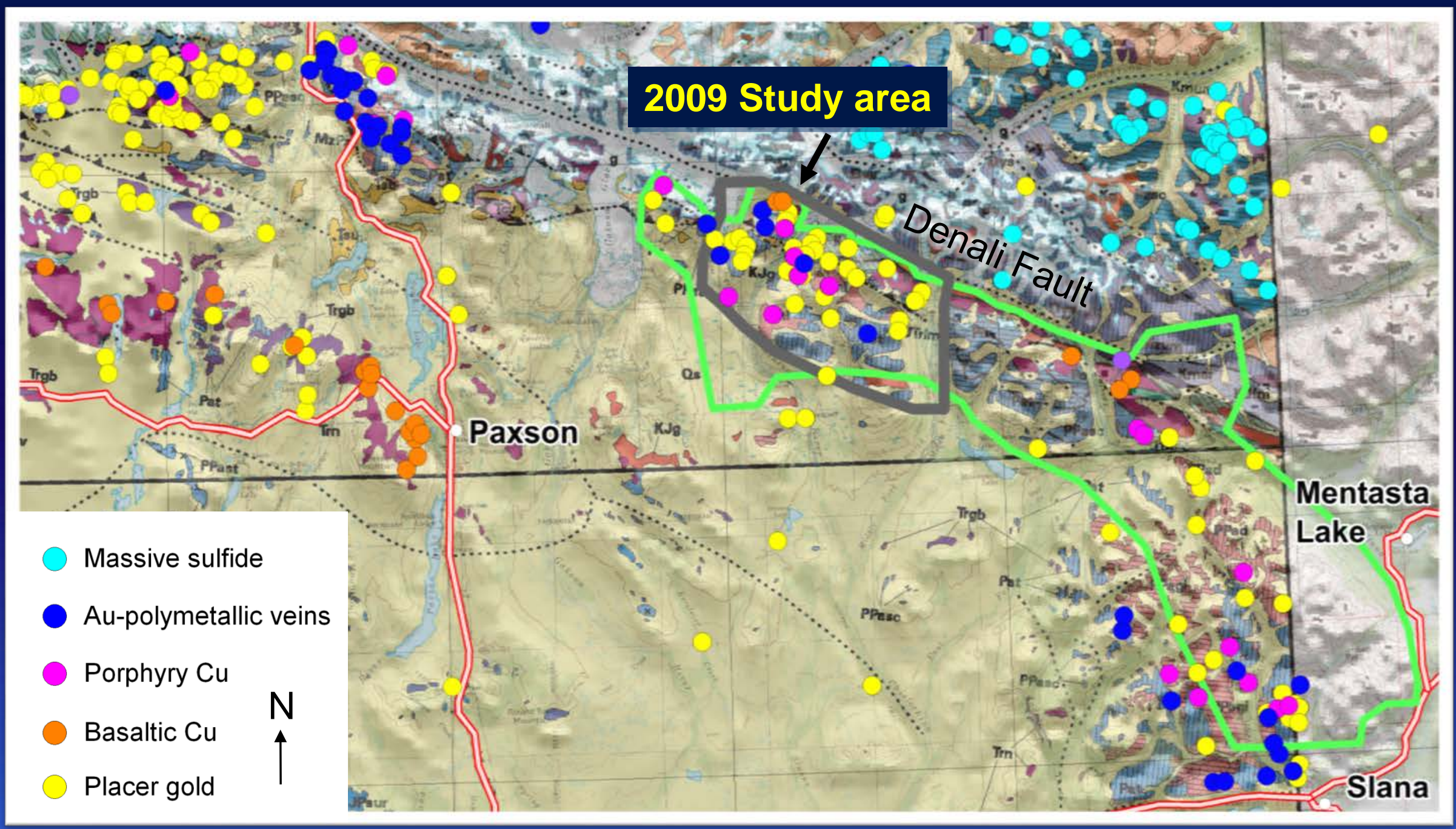

16 miles

Wilson et al., 1998 


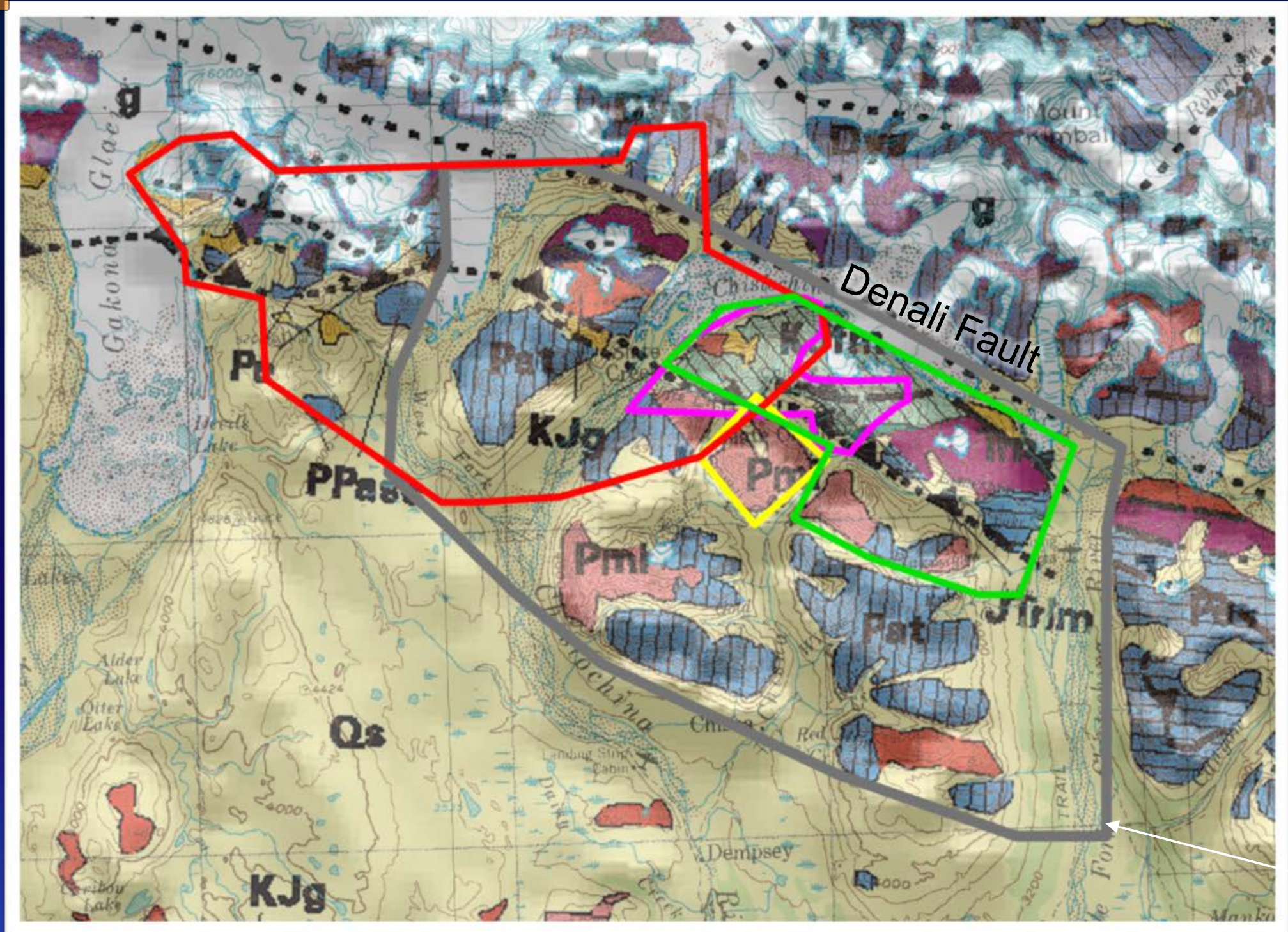

5 miles
Wilson et al., 1998
N

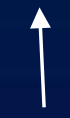

Rose, 1967

Matteson, 1973

Foley and Summers, 1990

Athey, 1999

STATEMAP Area $113 \mathrm{sq} \mathrm{mi}$ 


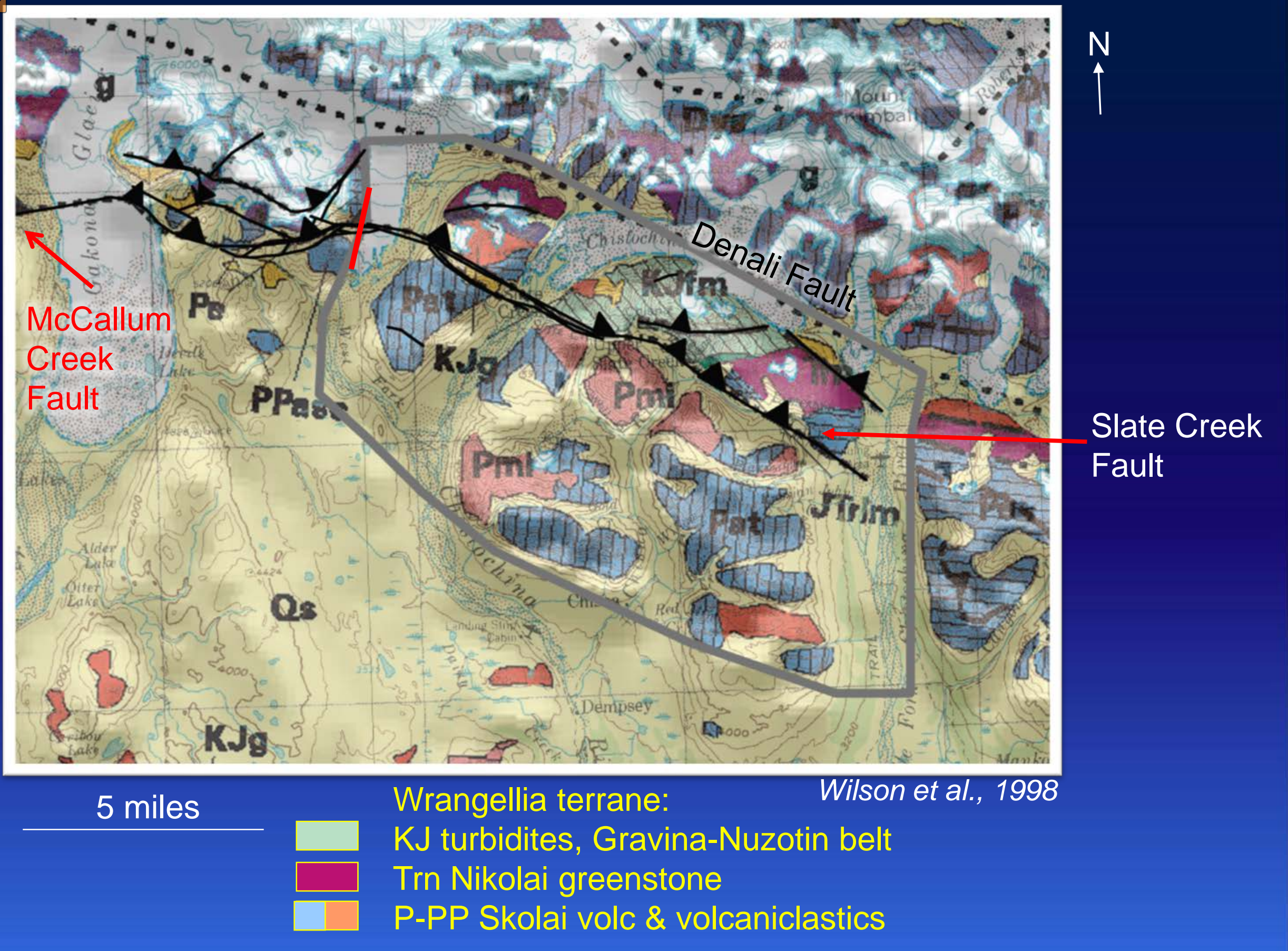




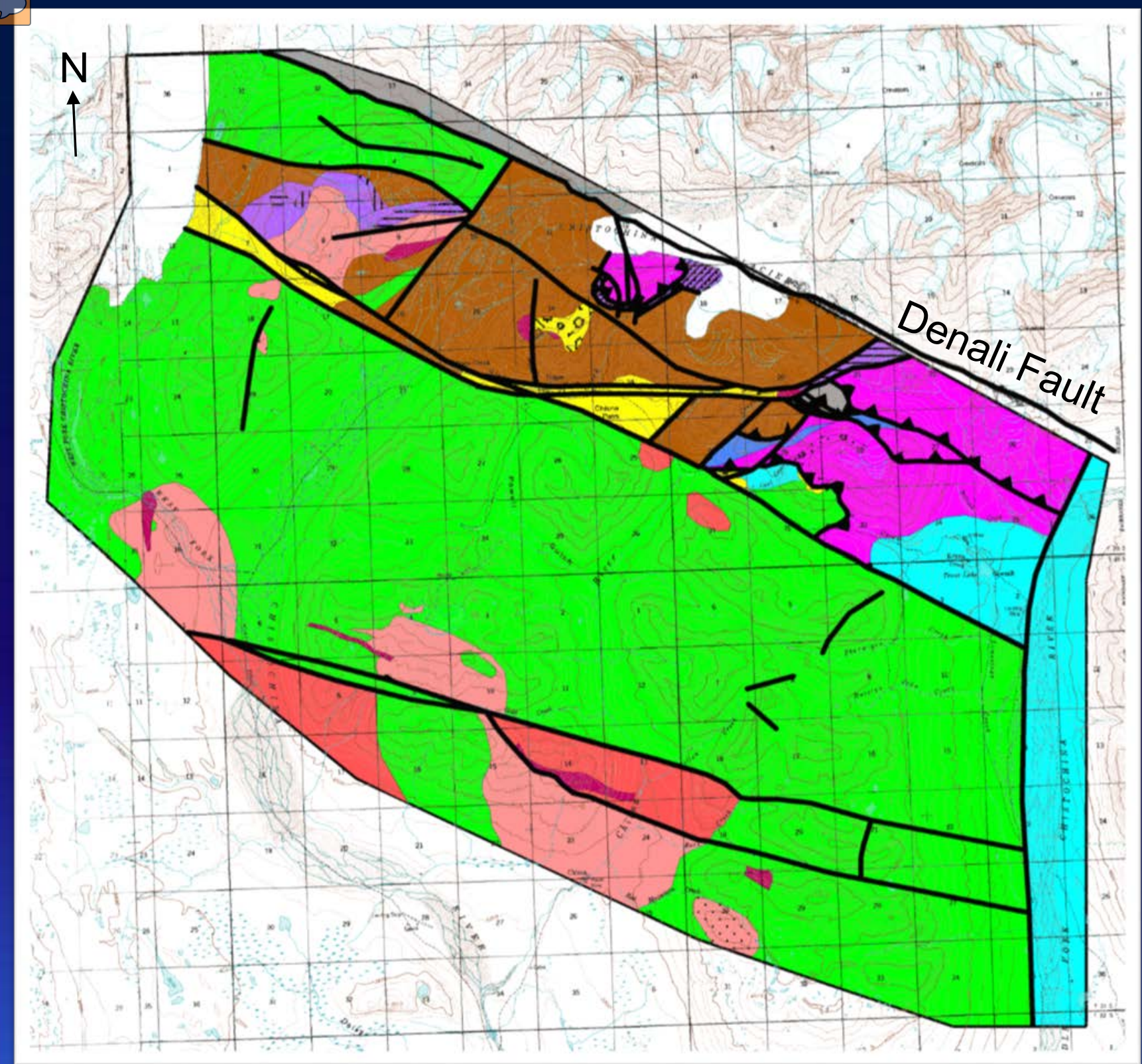

glacier

T seds and volcanics

Felsic intrusives

Intermediate intrusives

Mafic intrusives

Ultramafic intrusives

$\mathrm{KJ}$ argillte

Tr Chitistone Ims

Tr Nikolai greenstone

$P$ Eagle Creek Ims

P-PP Slana Spur

YTT metamorphics

DGGS's draft geologic map 


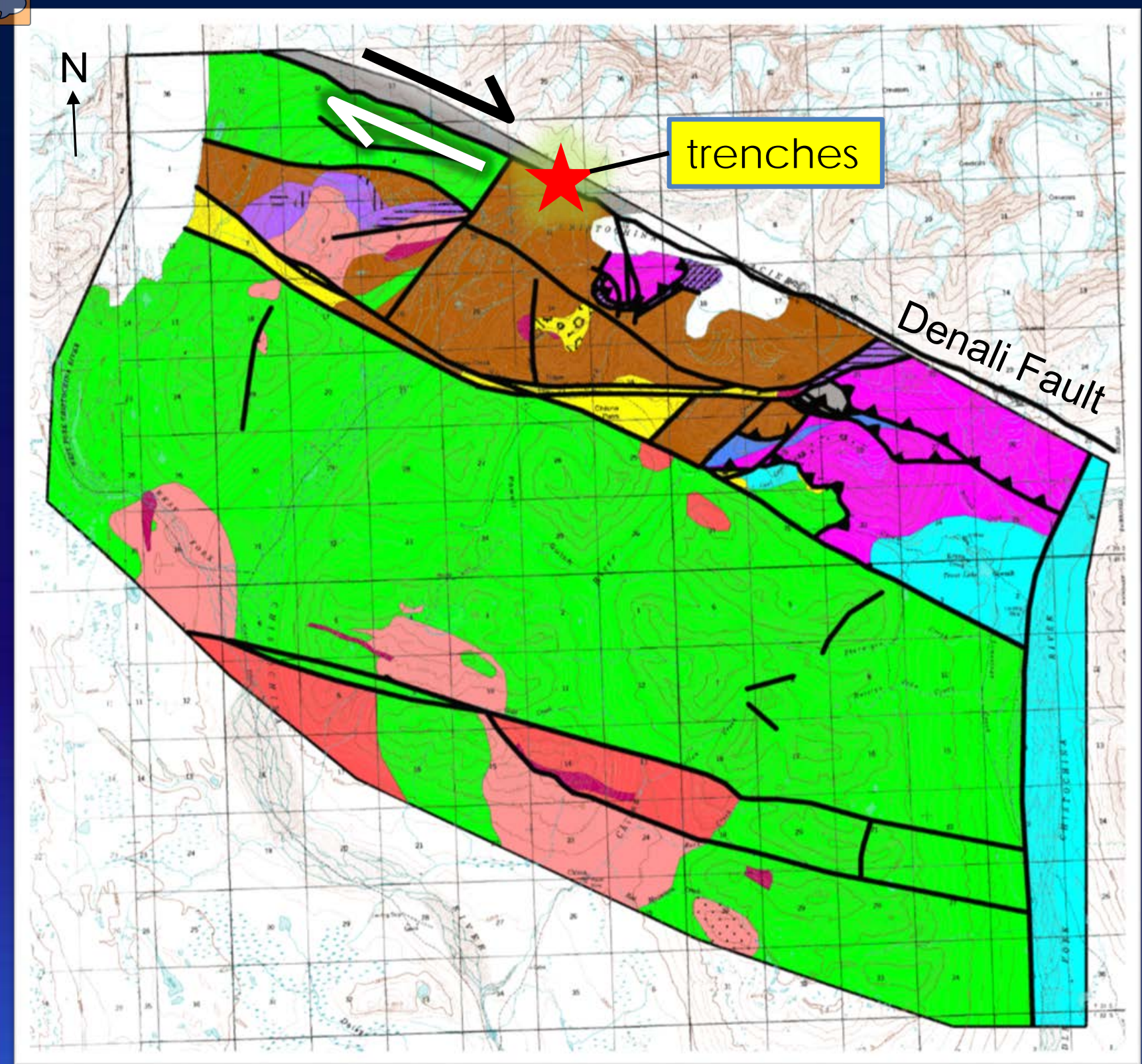

glacier

$T$ seds and volcanics

Felsic intrusives Intermediate intrusives Mafic intrusives Ultramafic intrusives $\mathrm{KJ}$ argillte Tr Chitistone Ims Tr Nikolai greenstone $P$ Eagle Creek Ims P-PP Slana Spur YTT metamorphics

Cooperative DGGS-USGS Denali Fault study 


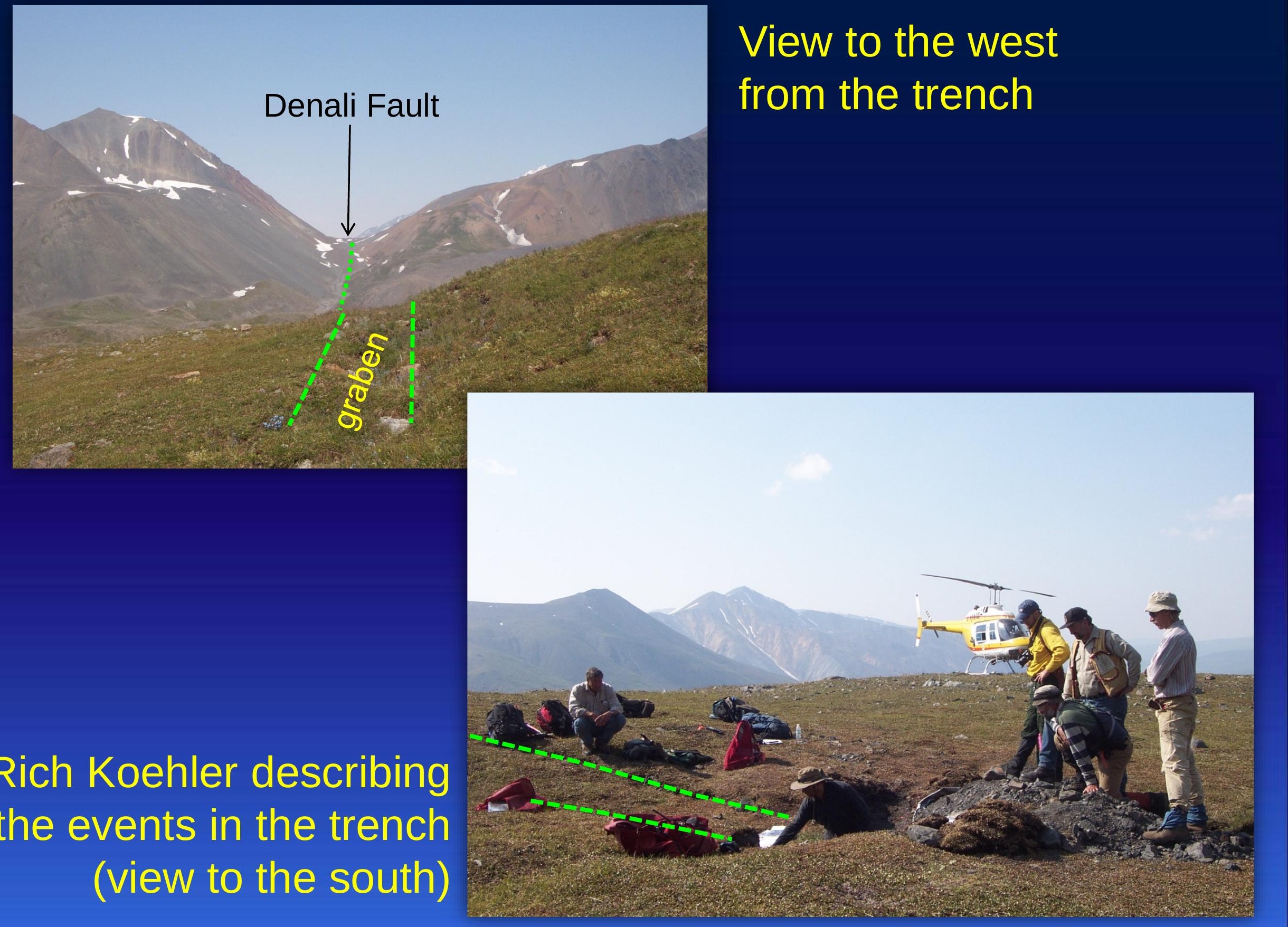

Rich Koehler describing the events in the trench (view to the south) from the trench 


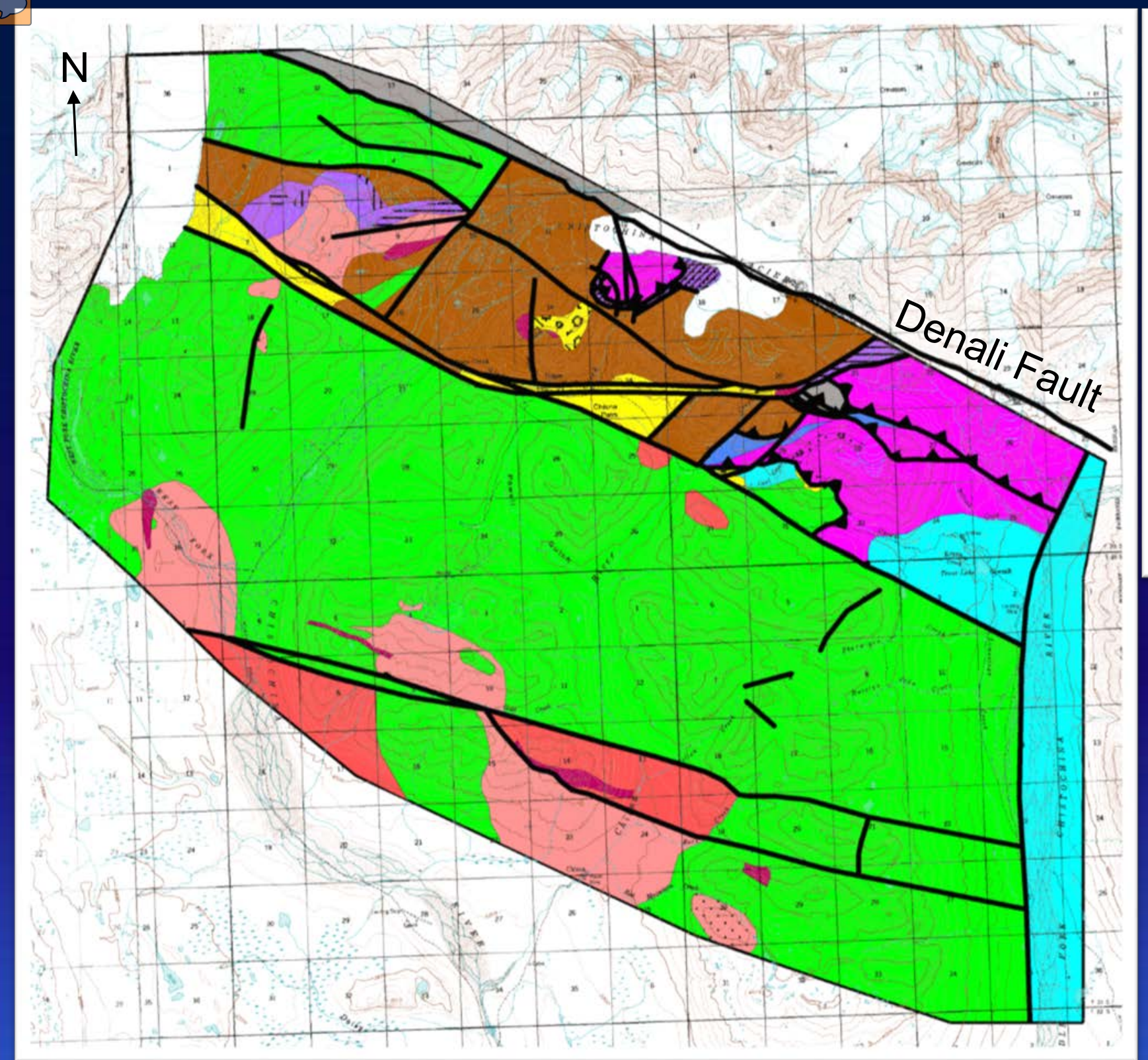

glacier

$T$ seds and volcanics

Felsic intrusives Intermediate intrusives Mafic intrusives Ultramafic intrusives $\mathrm{KJ}$ argillte

Tr Chitistone Ims Tr Nikolai greenstone $P$ Eagle Creek Ims P-PP Slana Spur YTT metamorphics

- Thrust and high angle faulting

- Long section of geologic history represented 


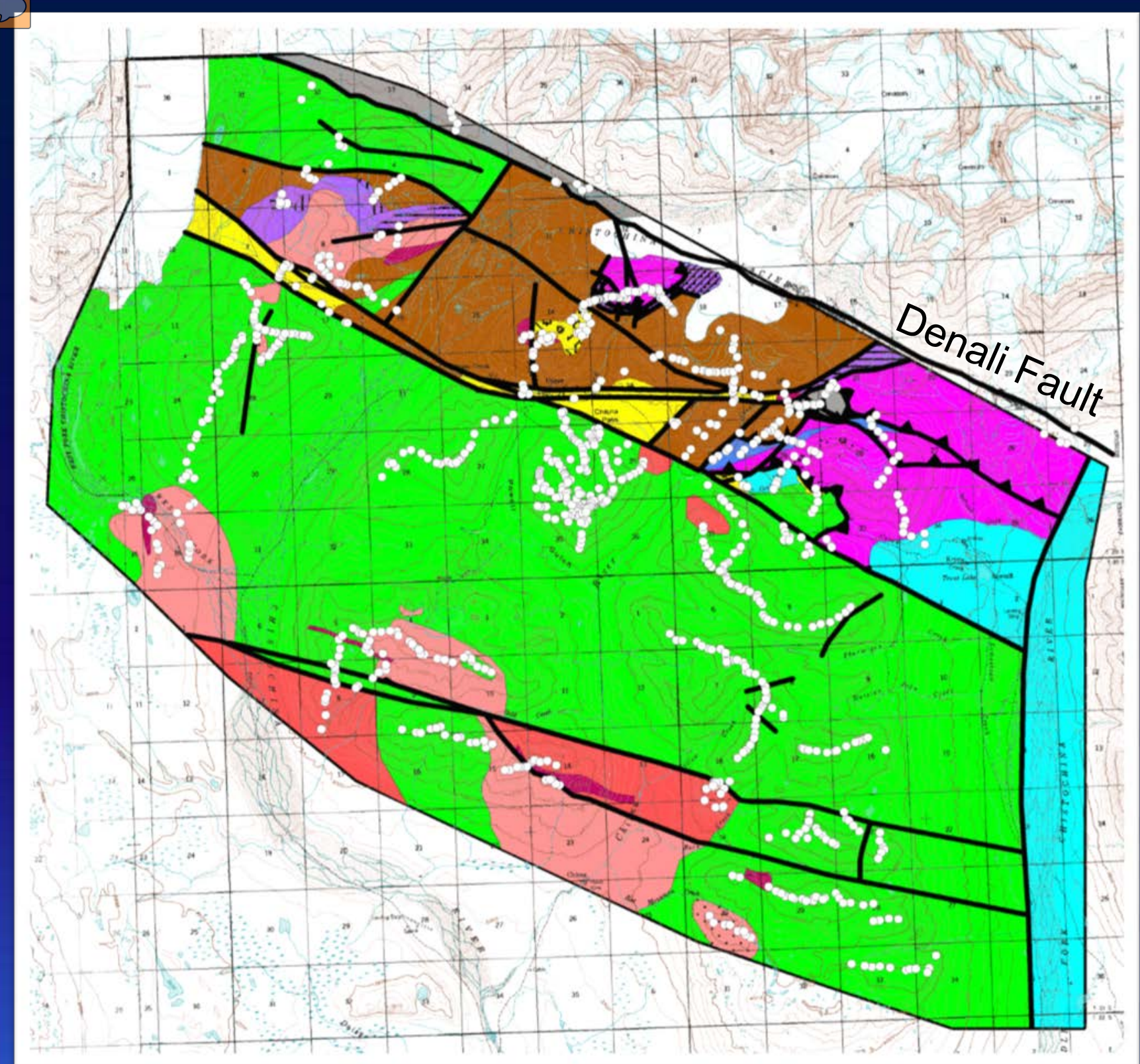

glacier

T seds and volcanics

Felsic intrusives Intermediate intrusives Mafic intrusives Ultramafic intrusives $\mathrm{KJ}$ argillte Tr Chitistone Ims Tr Nikolai greenstone $P$ Eagle Creek Ims P-PP Slana Spur YTT metamorphics

923 field stations from 2009 season and thesis work 


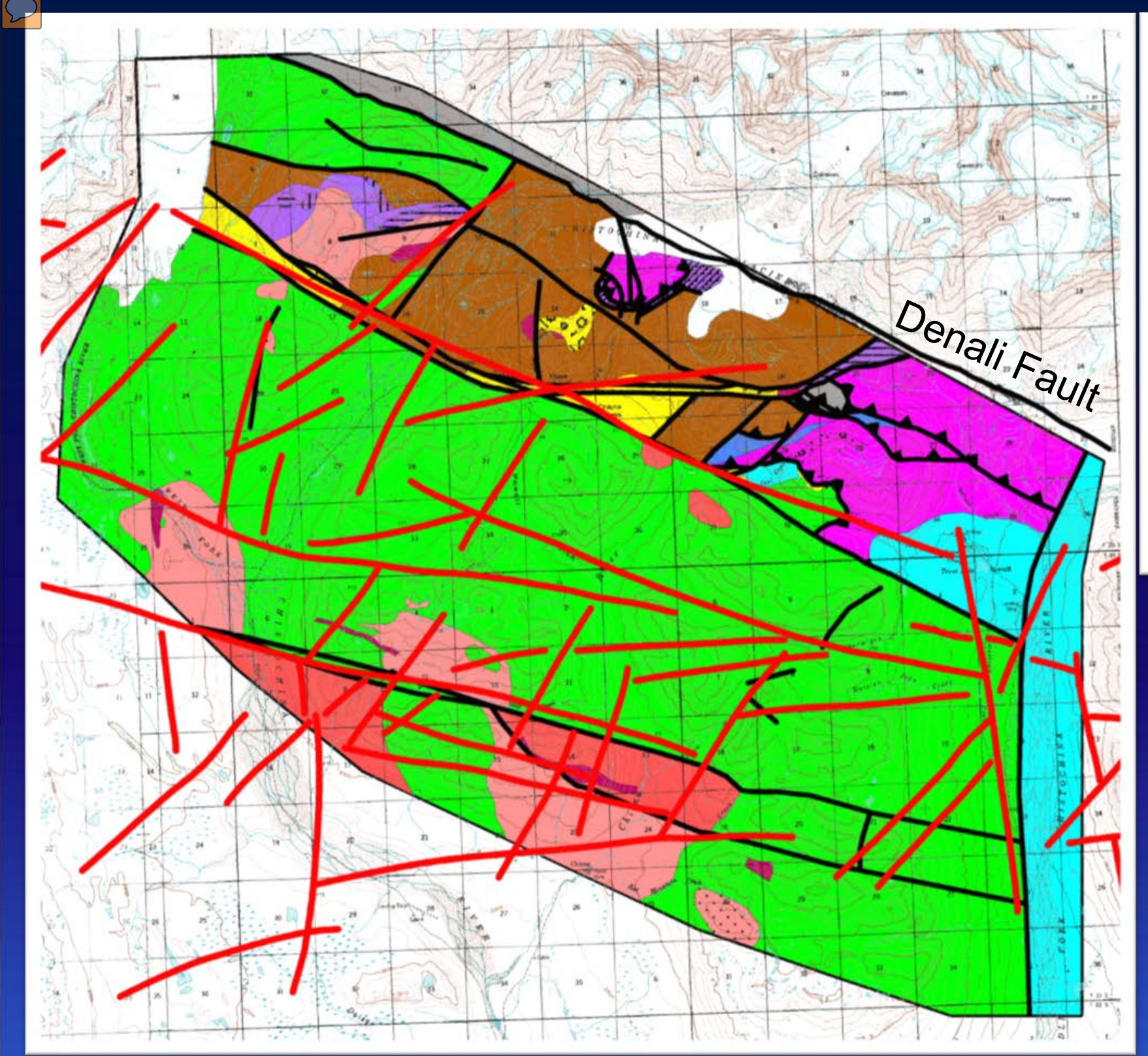

glacier

$T$ seds and volcanics

Felsic intrusives Intermediate intrusives Mafic intrusives Ultramafic intrusives $\mathrm{KJ}$ argillte $\operatorname{Tr}$ Chitistone Ims Tr Nikolai greenstone $P$ Eagle Creek Ims P-PP Slana Spur YTT metamorphics

Faults interpreted in the aeromagnetic and EM geophysical data (Burns and others, 2019) 


\section{Permian-Pennsylvanian Slana Spur Fm (Mankomen Group, originally called Chisna Fm by Mendenhall [1905]):}

Volcaniclastics, tuffs, andesite flows, conglomerate, thin limestones, volcanic sedimentary rocks (basaltic to rhyolitic composition volcanics)

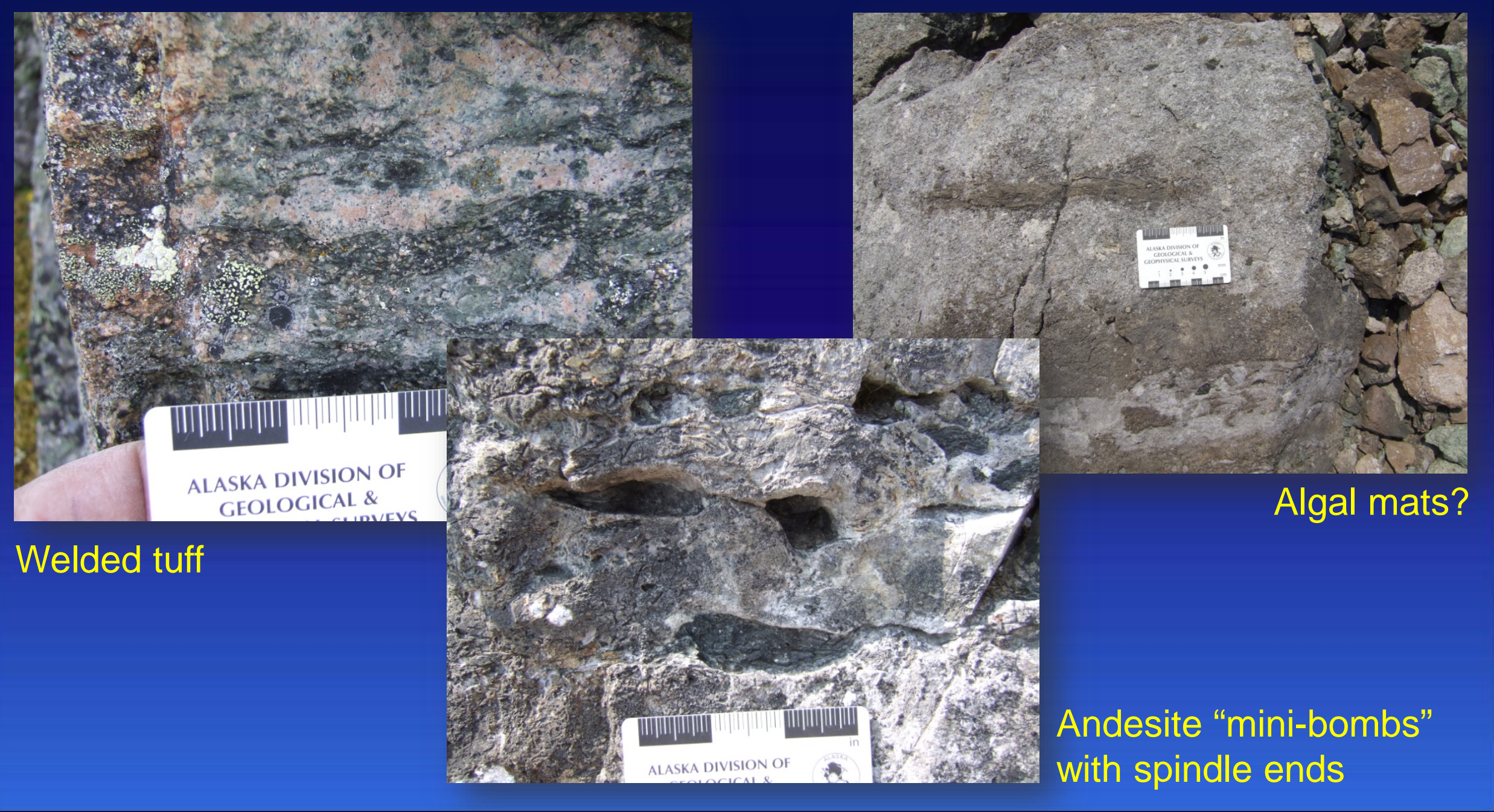




\section{Pennsylvanian Tetelna Volcanics (Mendenhall, 1905):}

Massive andesite flows with minor volcaniclastics
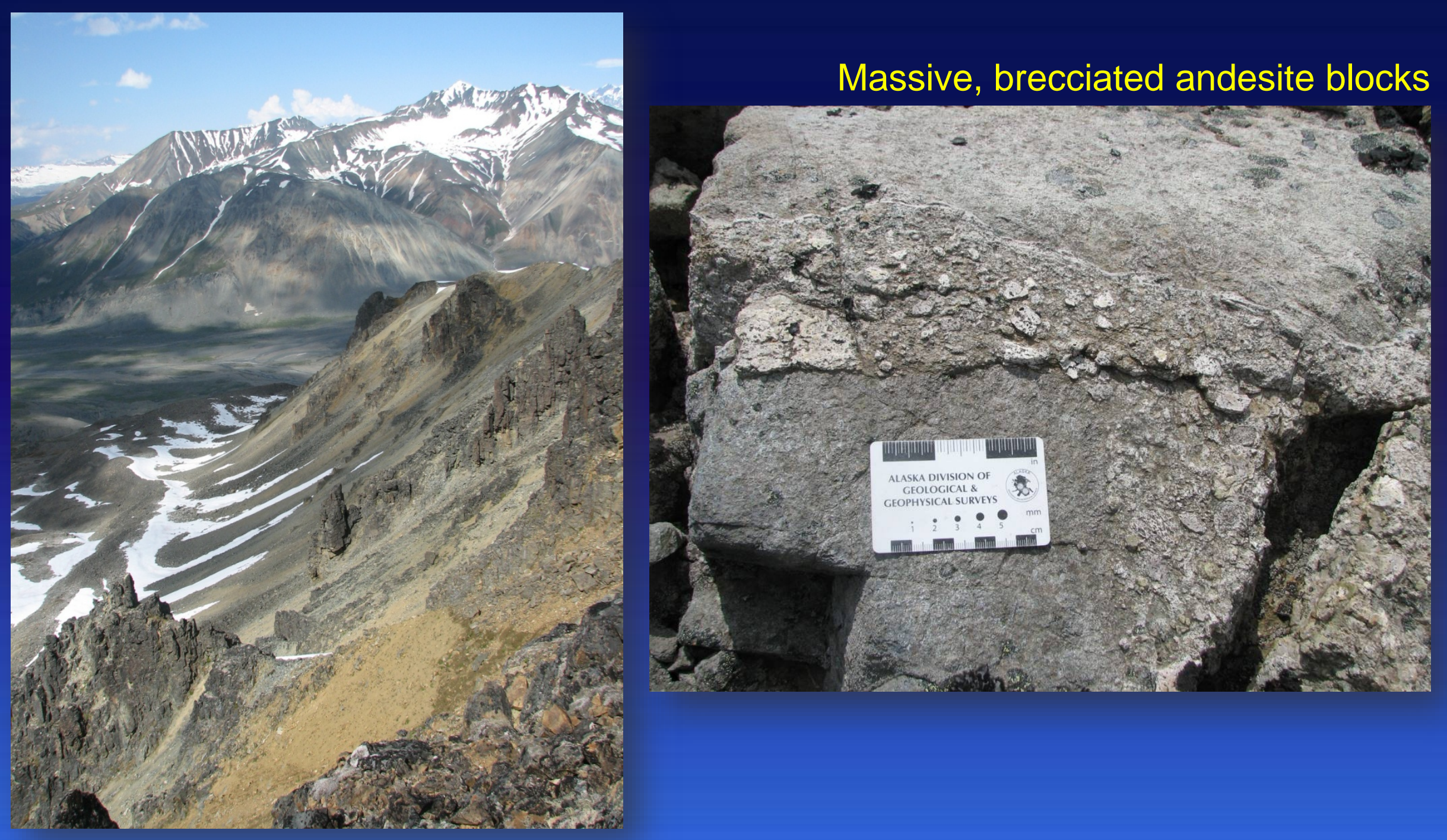


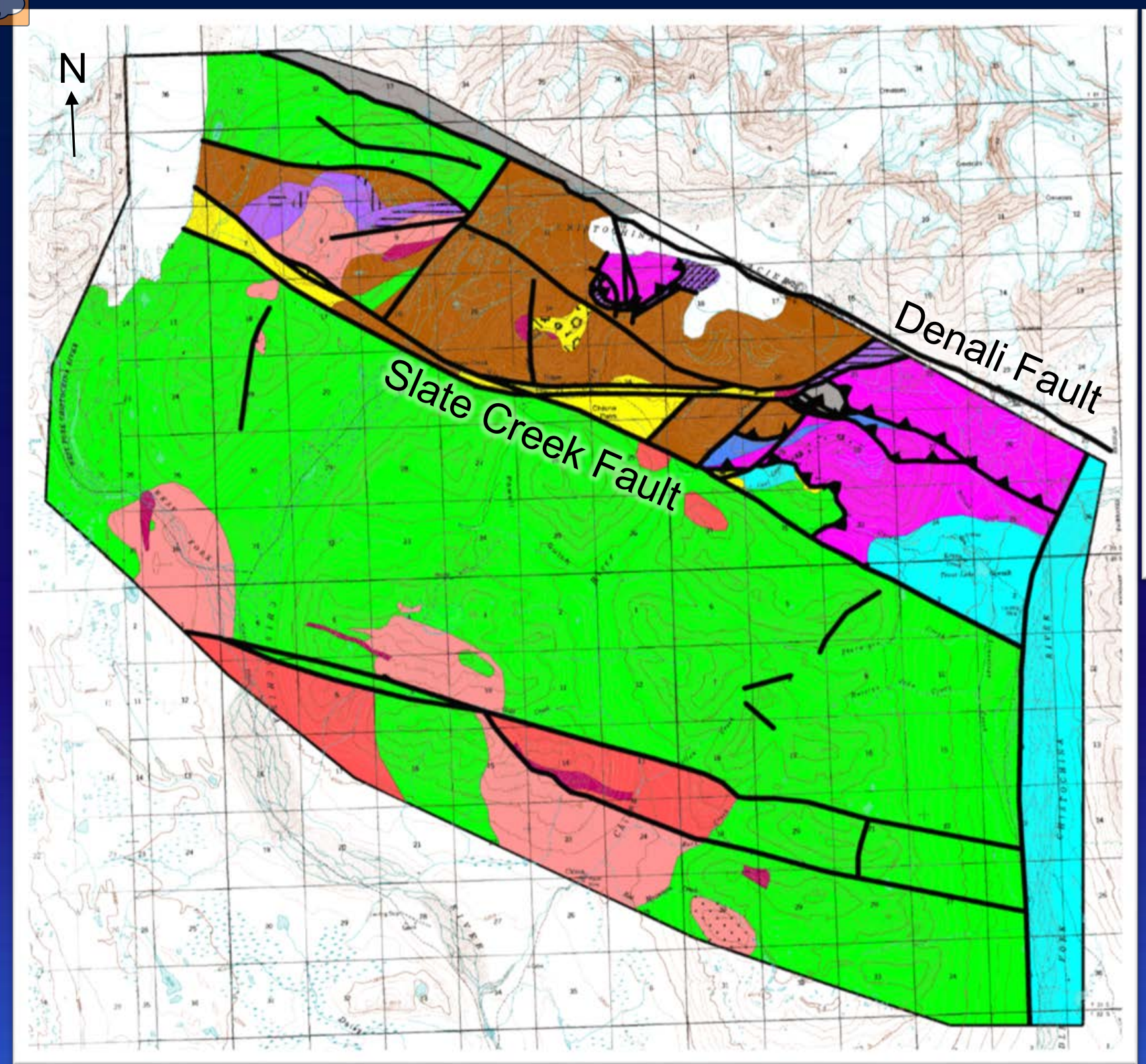

glacier

$T$ seds and volcanics

Felsic intrusives Intermediate intrusives Mafic intrusives Ultramafic intrusives $\mathrm{KJ}$ argillte Tr Chitistone Ims Tr Nikolai greenstone $P$ Eagle Creek Ims P-PP Slana Spur YTT metamorphics

- Shallow northdipping thrusts

- Thrust plate thickens and deepens to NW 


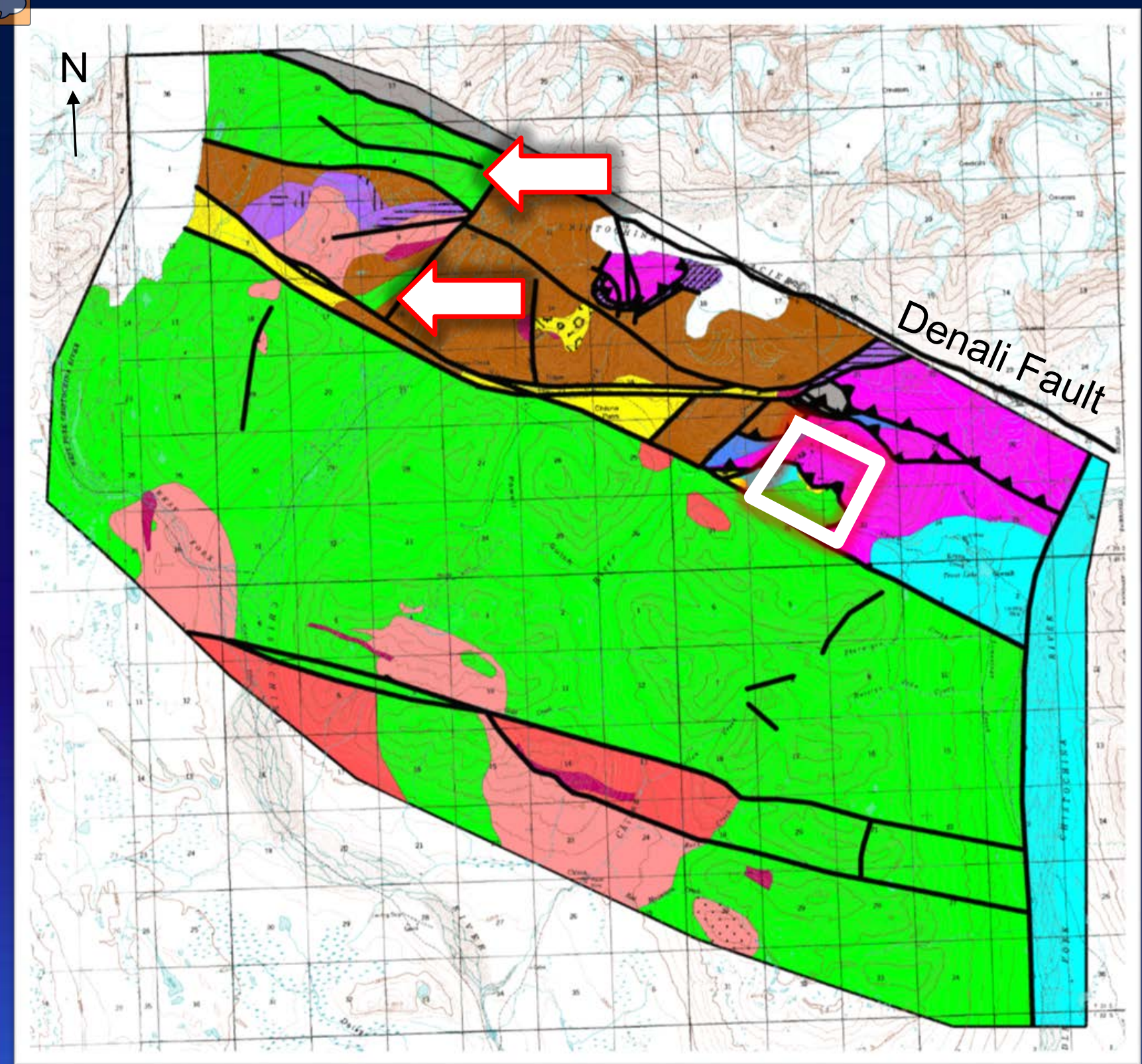

glacier

$T$ seds and volcanics

Felsic intrusives Intermediate intrusives Mafic intrusives Ultramafic intrusives $\mathrm{KJ}$ argillte Tr Chitistone Ims Tr Nikolai greenstone $P$ Eagle Creek Ims P-PP Slana Spur YTT metamorphics

- Slana Spur north of Slate Creek

- Eagle Ck Fm 


\section{Early Permian Eagle Creek Fm (Mankomen Group):}

Limestone, argillite, graywacke
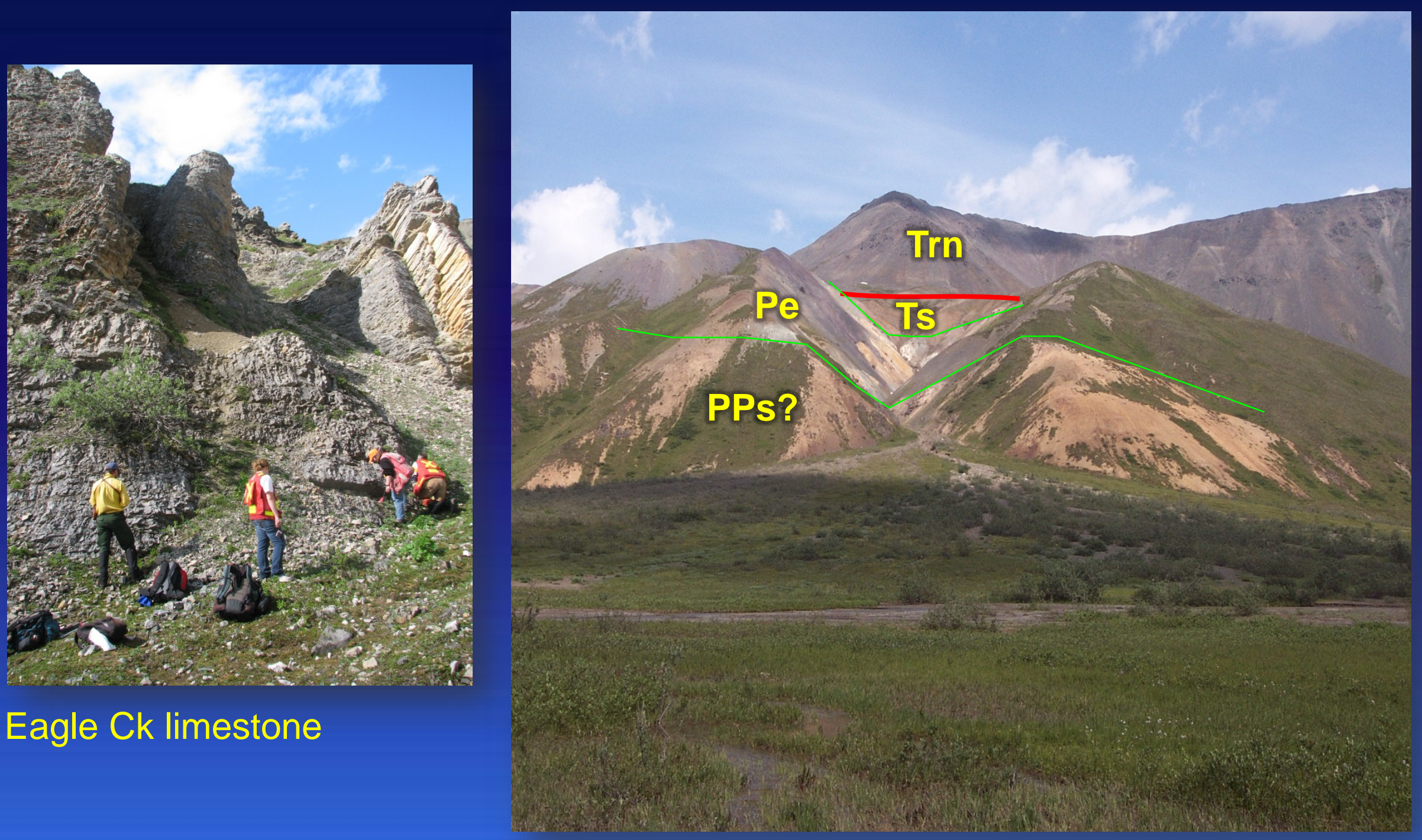

Eagle Ck limestone 


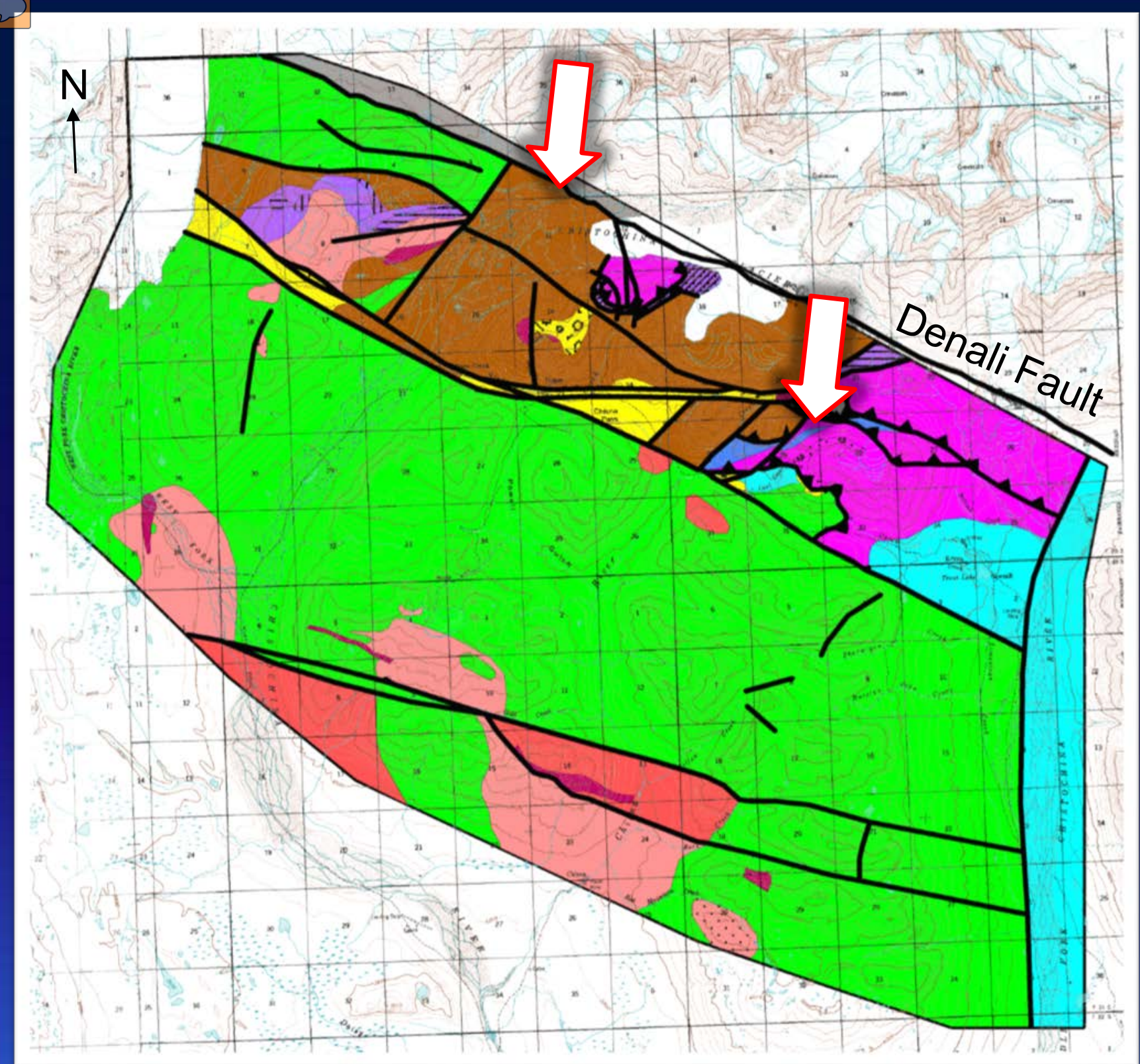

glacier

$T$ seds and volcanics

Felsic intrusives Intermediate intrusives Mafic intrusives Ultramafic intrusives $\mathrm{KJ}$ argillte Tr Chitistone Ims Tr Nikolai greenstone $P$ Eagle Creek Ims P-PP Slana Spur YTT metamorphics 


\section{Late Triassic Nikolai Greenstone and Chitistone Limestone:}

Weakly metamorphosed basalt, Chitistone limestone, minor siltstone and conglomerate

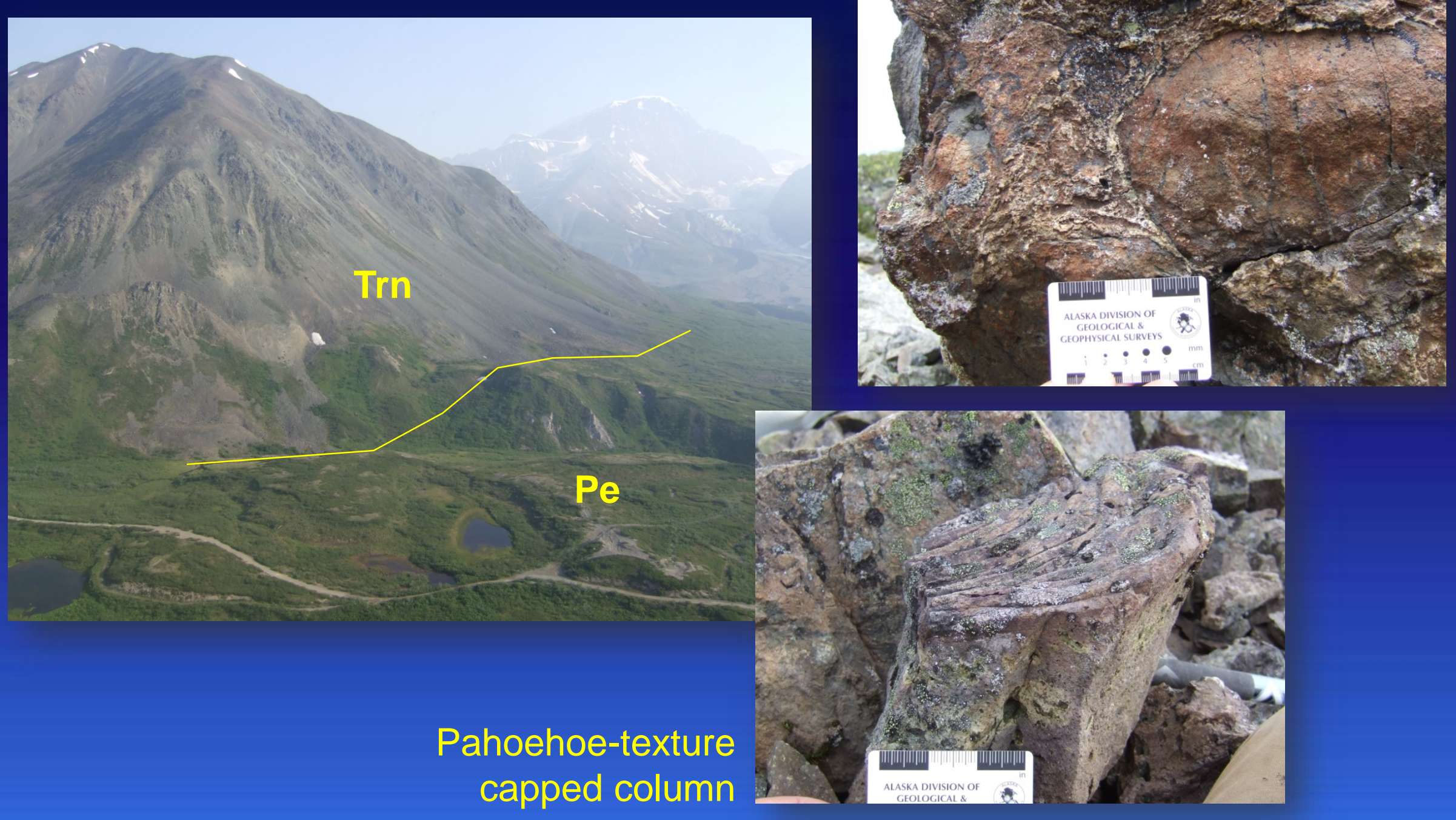




\section{Overturned folding}

\section{Cretaceous-Jurassic argillte/phyllite}
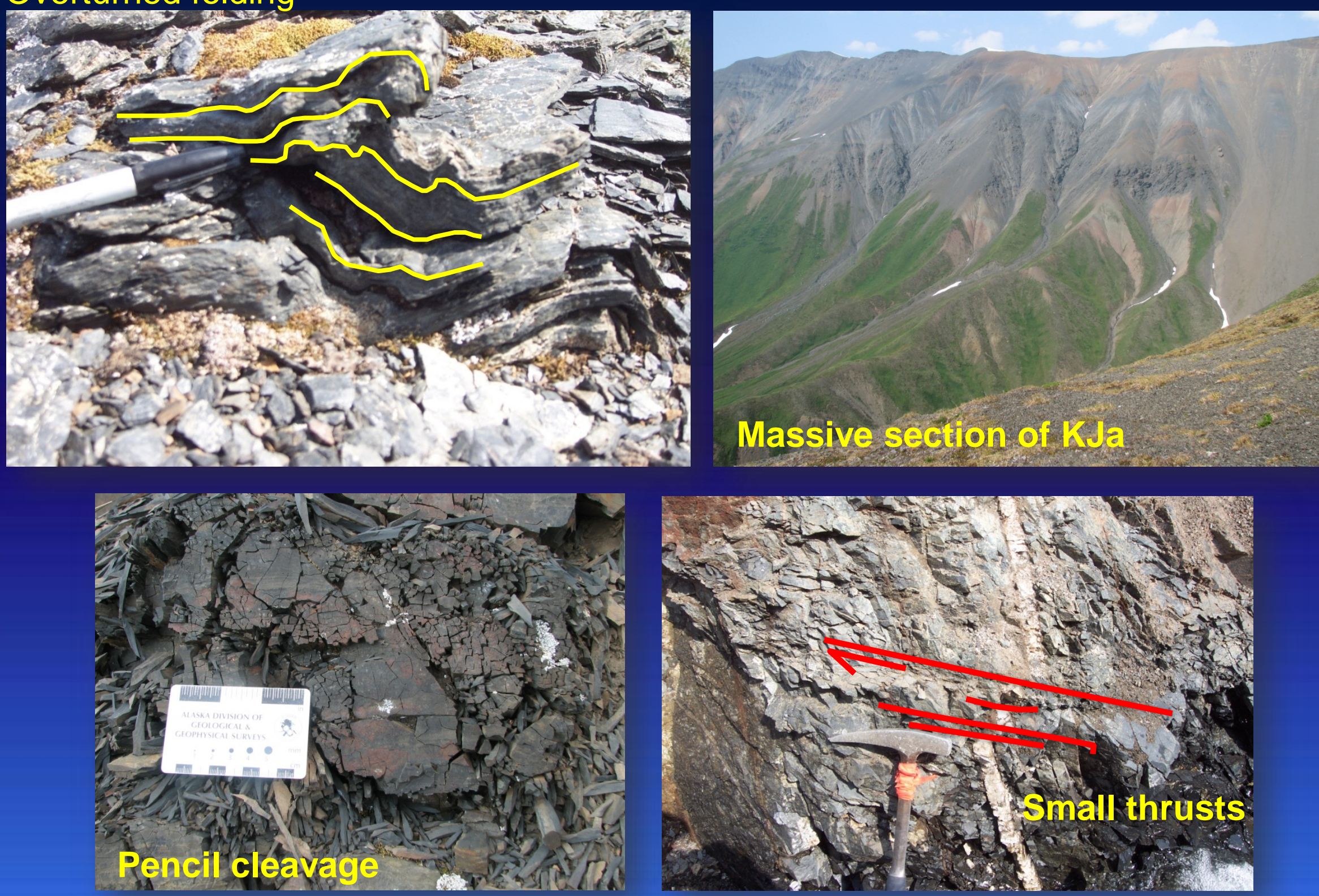


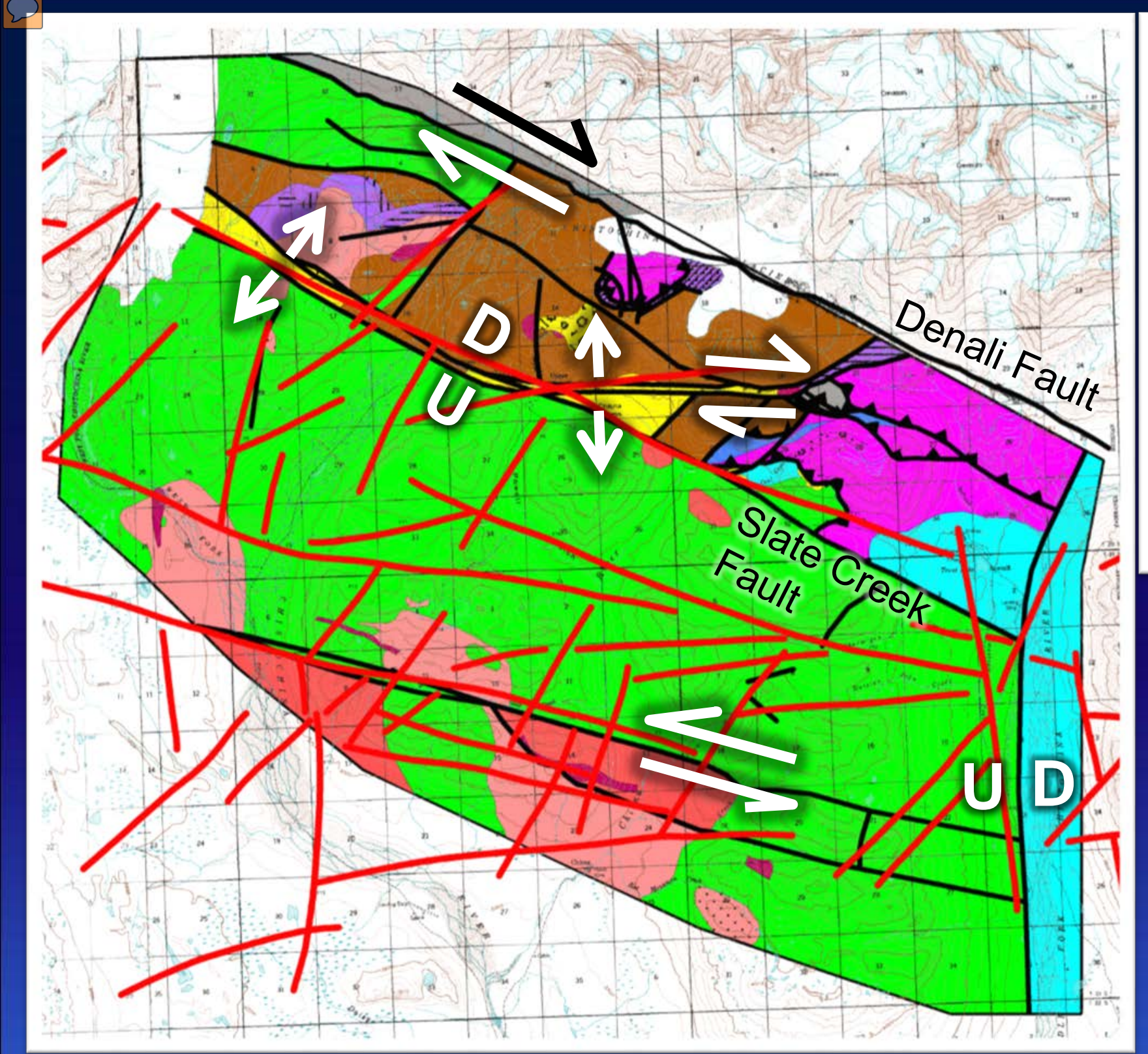

glacier

T seds and volcanics

Felsic intrusives Intermediate intrusives Mafic intrusives Ultramafic intrusives $\mathrm{KJ}$ argillte $\operatorname{Tr}$ Chitistone Ims Tr Nikolai greenstone $P$ Eagle Creek Ims P-PP Slana Spur YTT metamorphics

Faults interpreted in the aeromagnetic and EM geophysical data (Burns and others, 2019) 

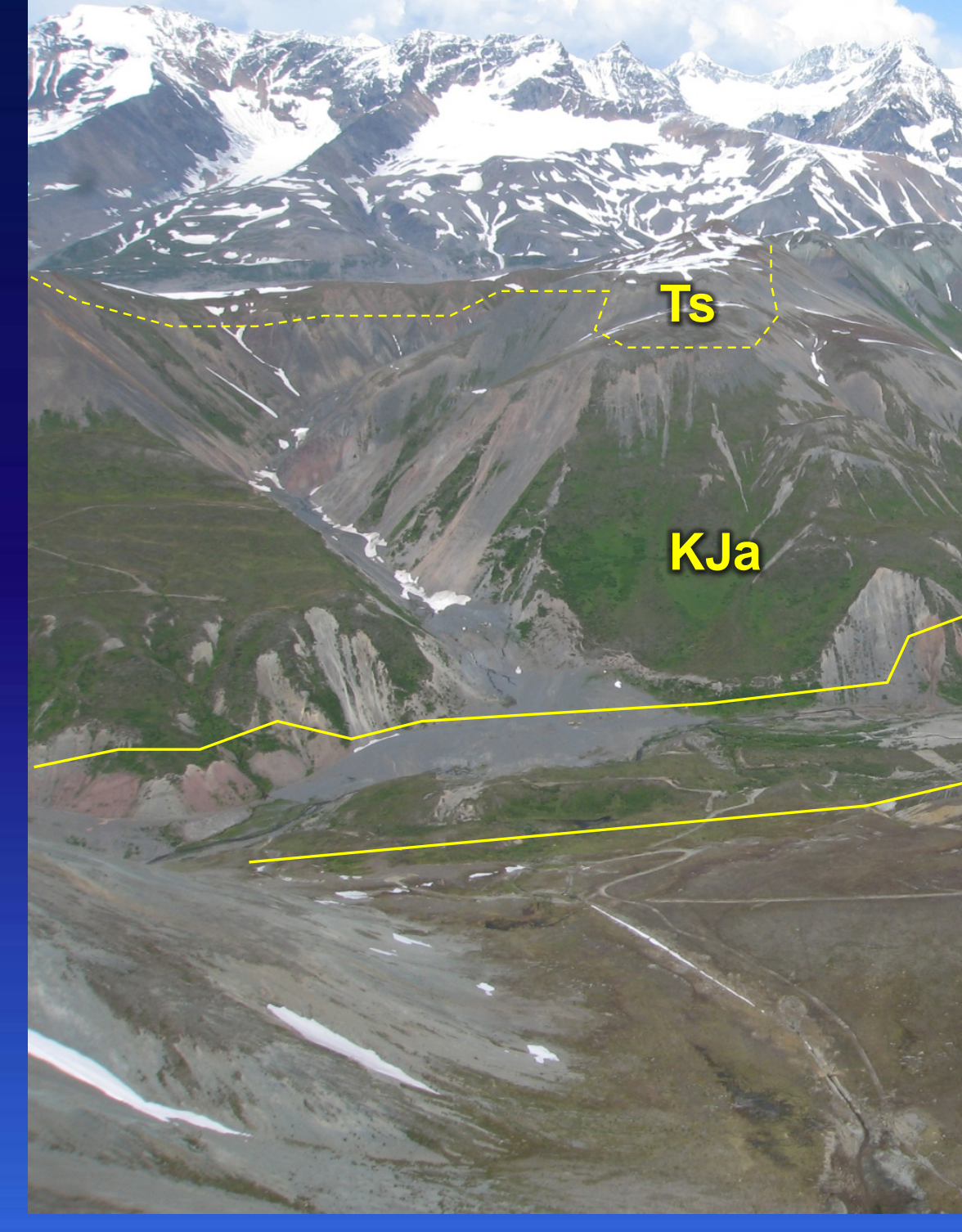

ato

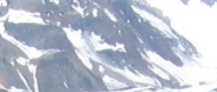

$+$
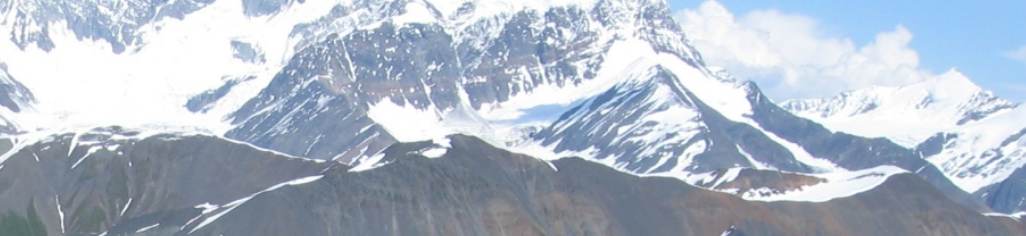

$-1+--$

il

1.

Ts

PPs

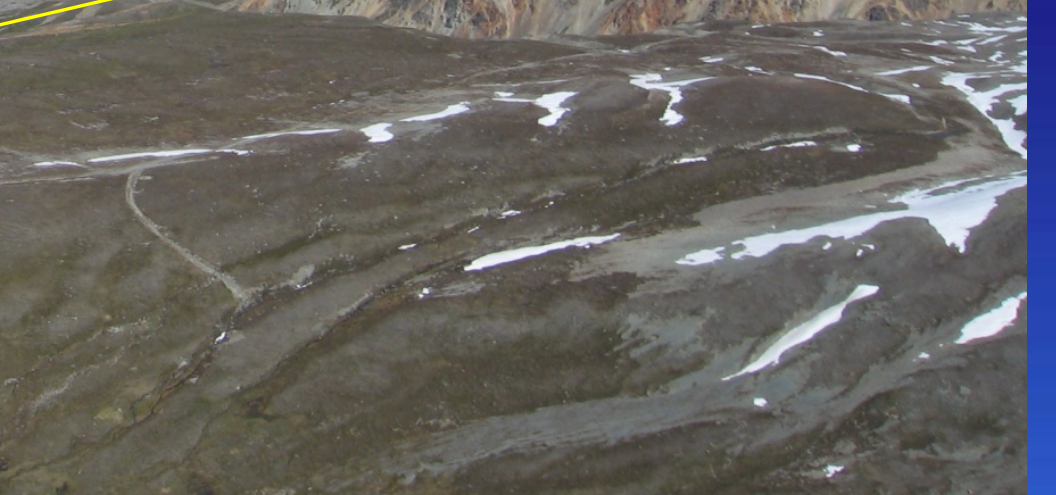

Slate Creek, looking northeast 


\section{Tertiary (Miocene-Eocene) Gakona Fm?}

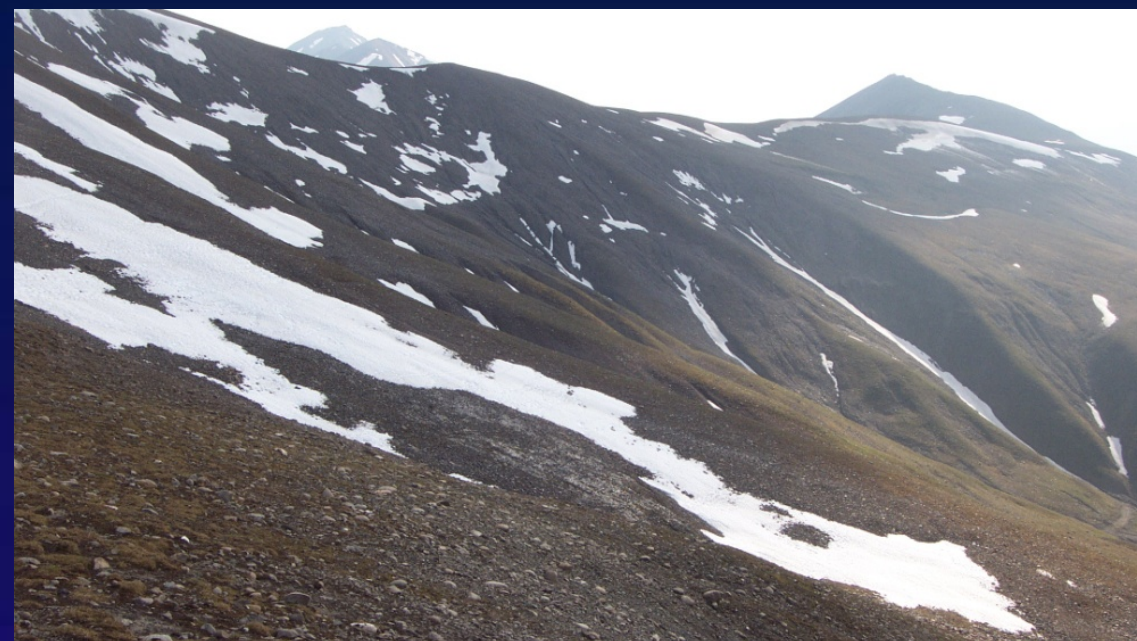

Tc on north side of Miller Gulch

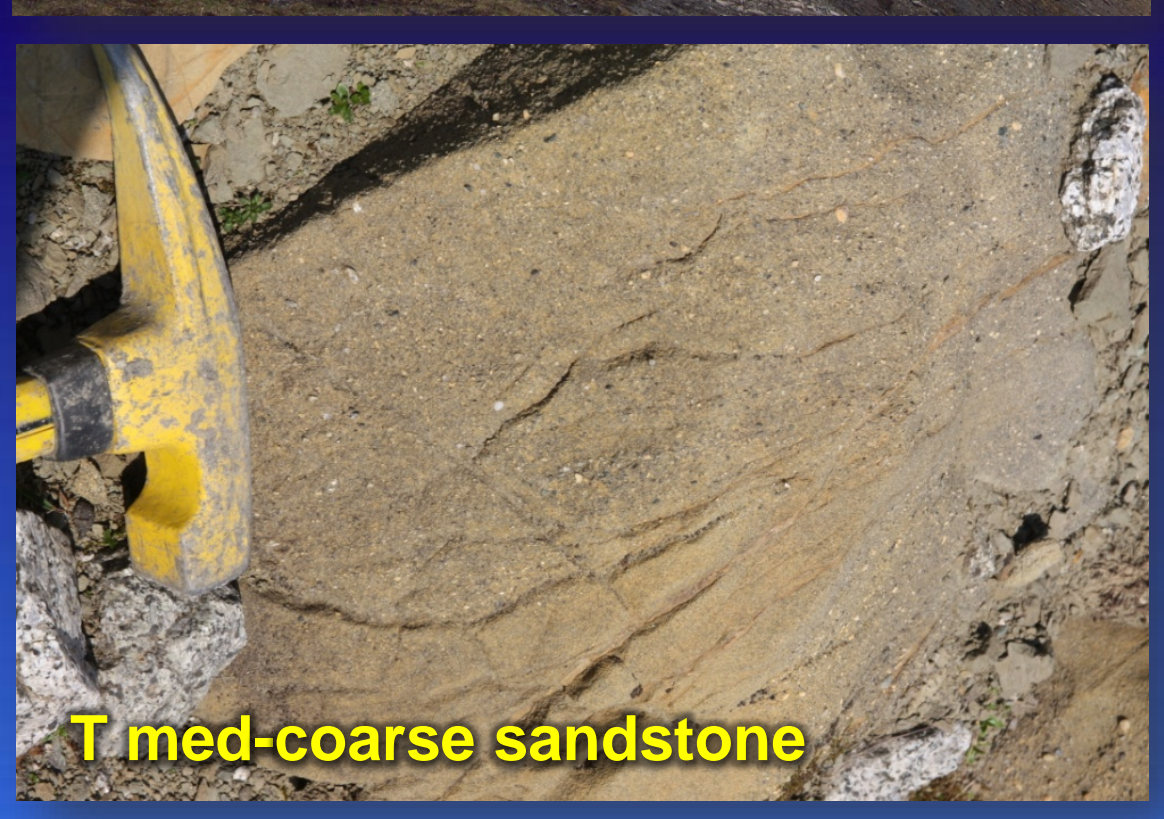

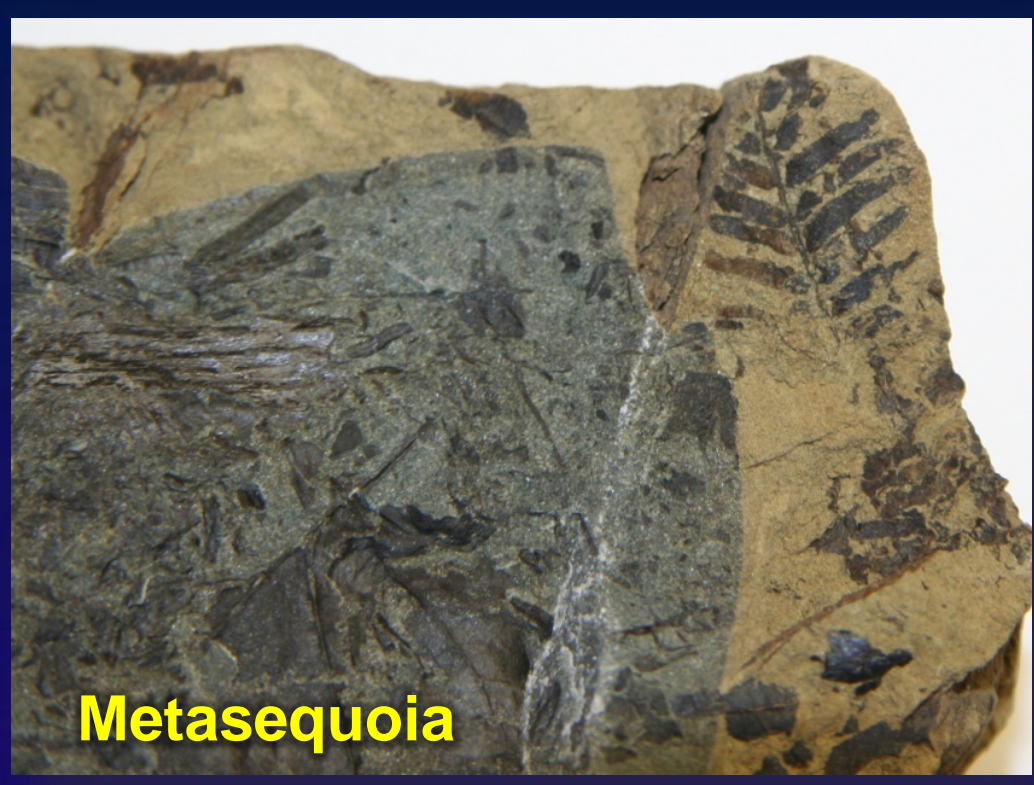

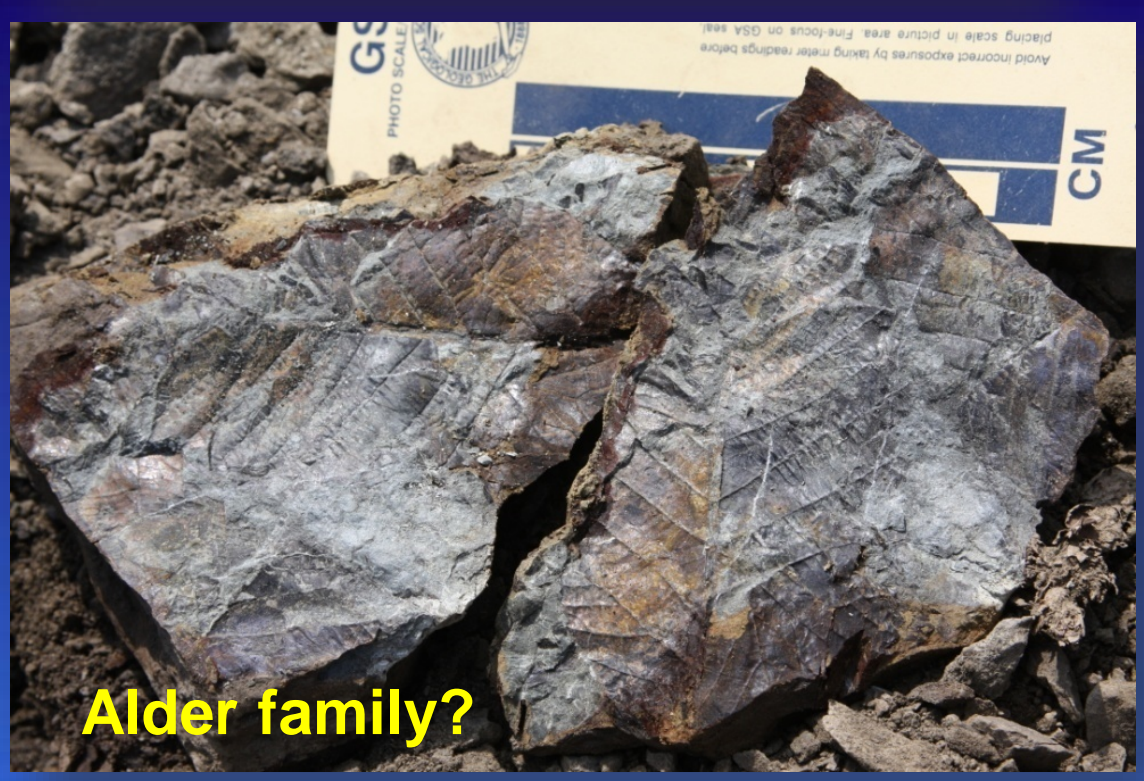




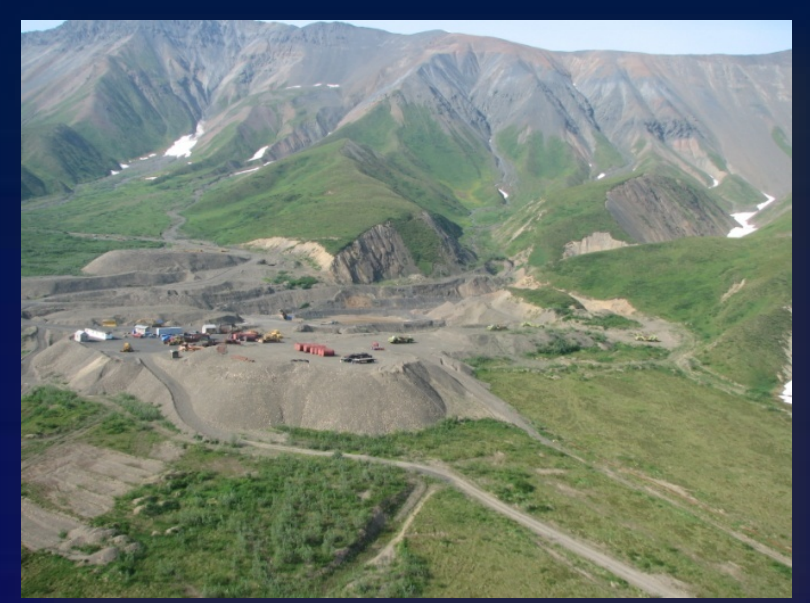

\section{Sources of Gold}

Roundwash concentrate (non-magnetic fraction): $3.31 \mathrm{oz} / \mathrm{T}$ gold (Bittenbender et al., 2007)

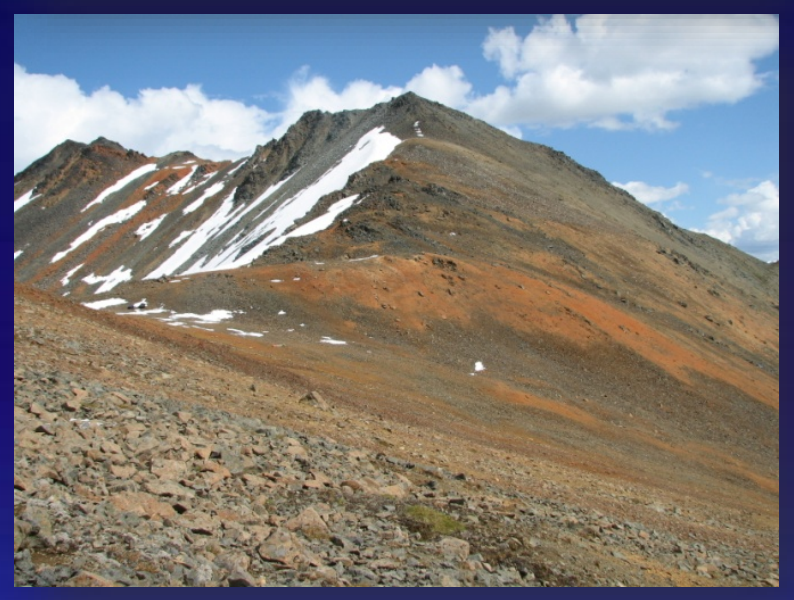

Quartz-pyrite gossan in Slana Spur Fm.: Up to $0.72 \mathrm{oz} / \mathrm{T}$ gold (Athey, 1999); associated with felsic volcanics

Hydrothermally altered argillite: concentrate $0.008 \mathrm{oz} / \mathrm{T}$ gold tabled tailings $0.005 \mathrm{oz} / \mathrm{T}$ gold (Foley and Summers, 1990) 


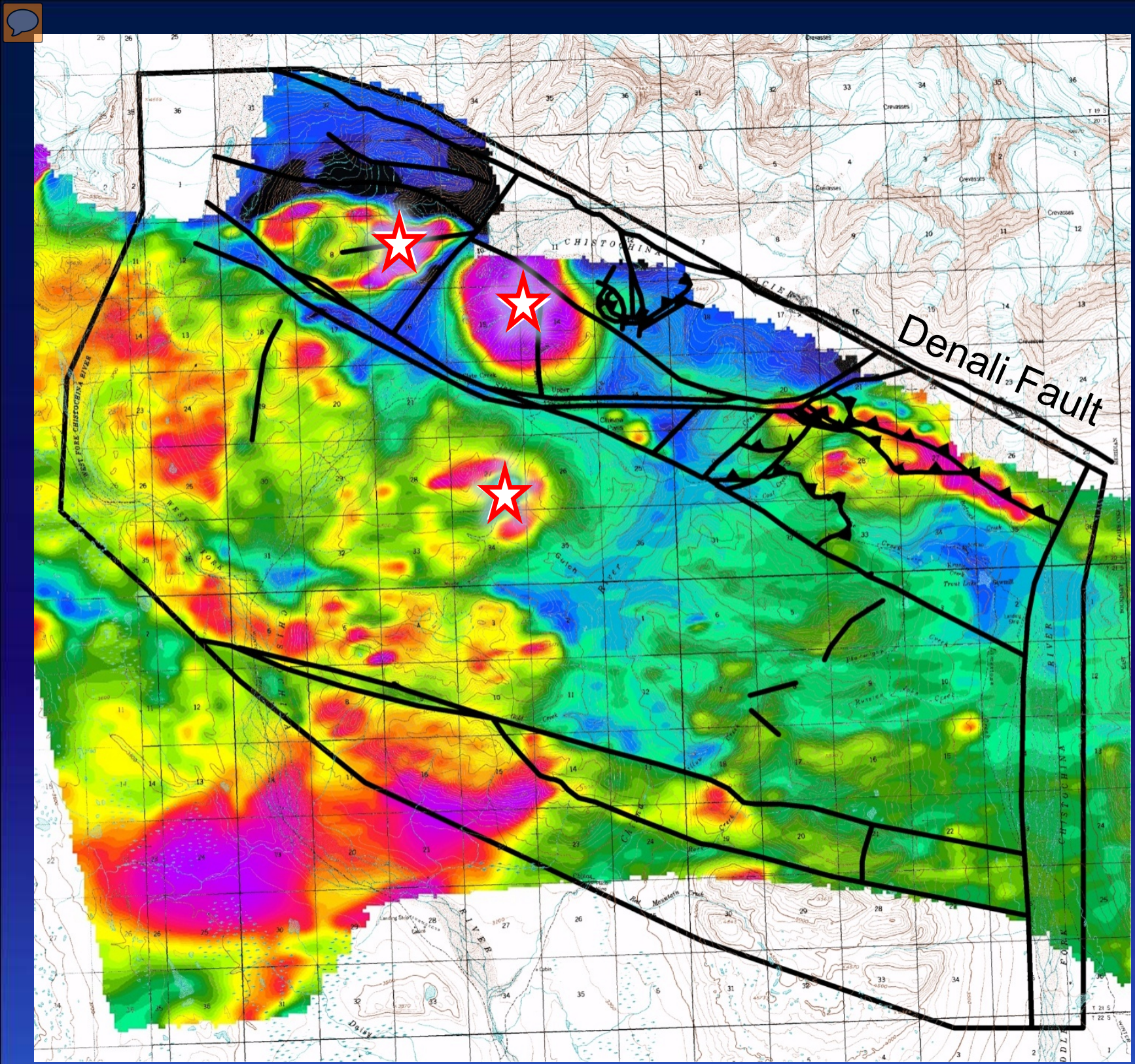

Total field magnetics

Purple-red - highs Blue-black - lows

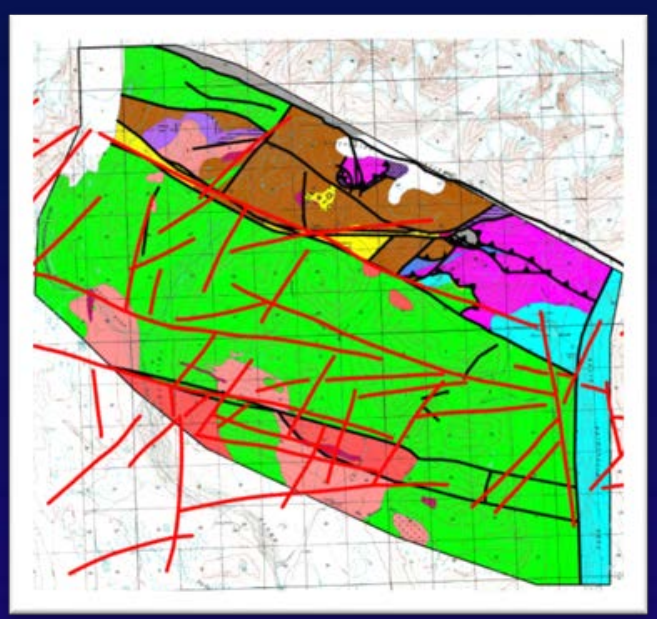

5 miles 


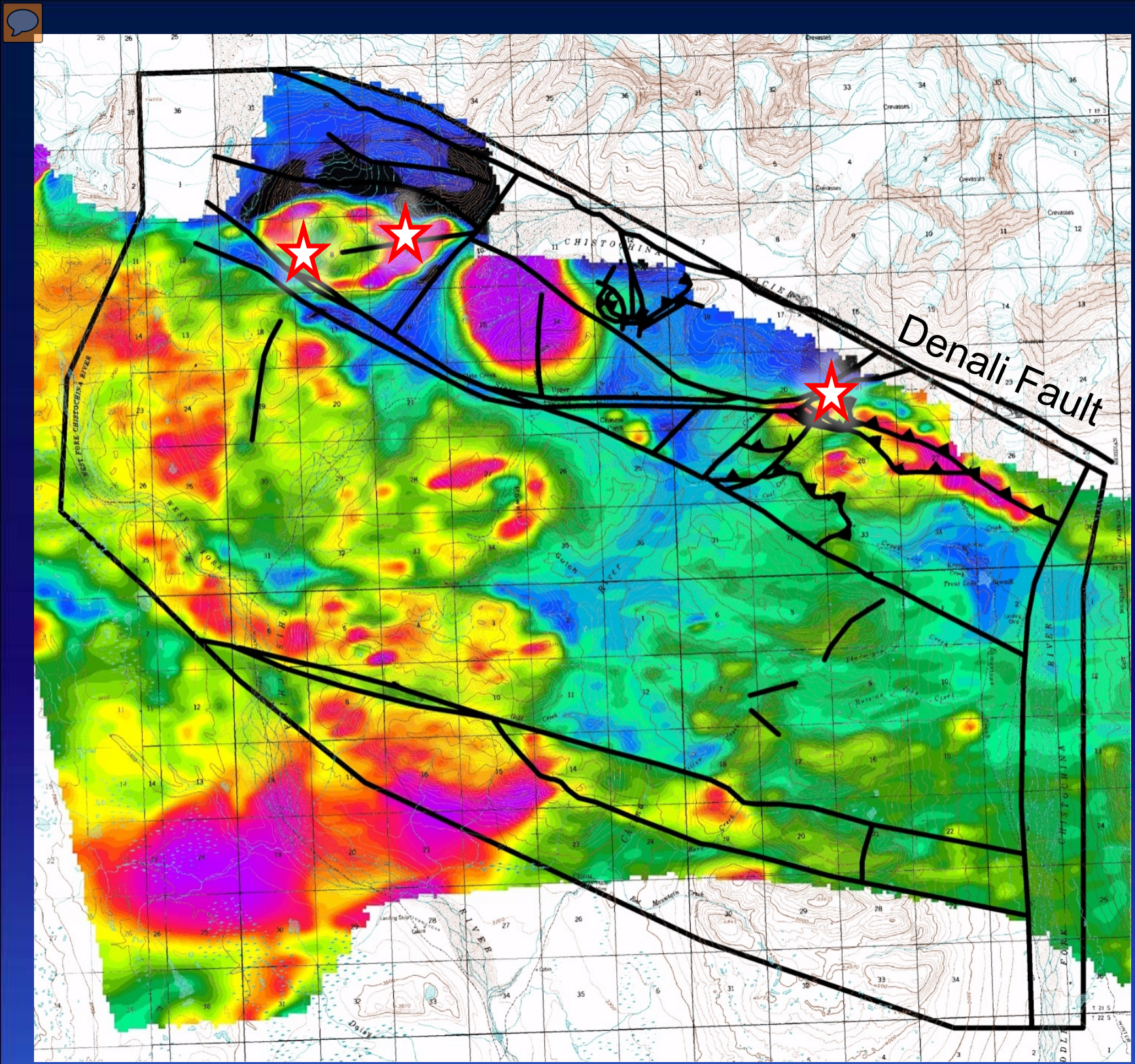

Total field magnetics

Purple-red - highs Blue-black - lows

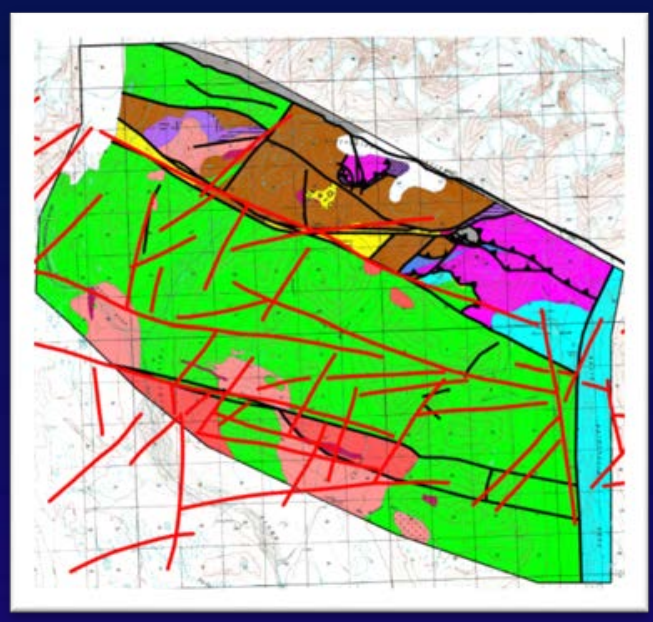

5 miles

Ar-Ar ages:

$119-123 \mathrm{Ma}$ (Bittenbender et al., 2007) 


\section{Cretaceous mafic and ultramafic rocks}
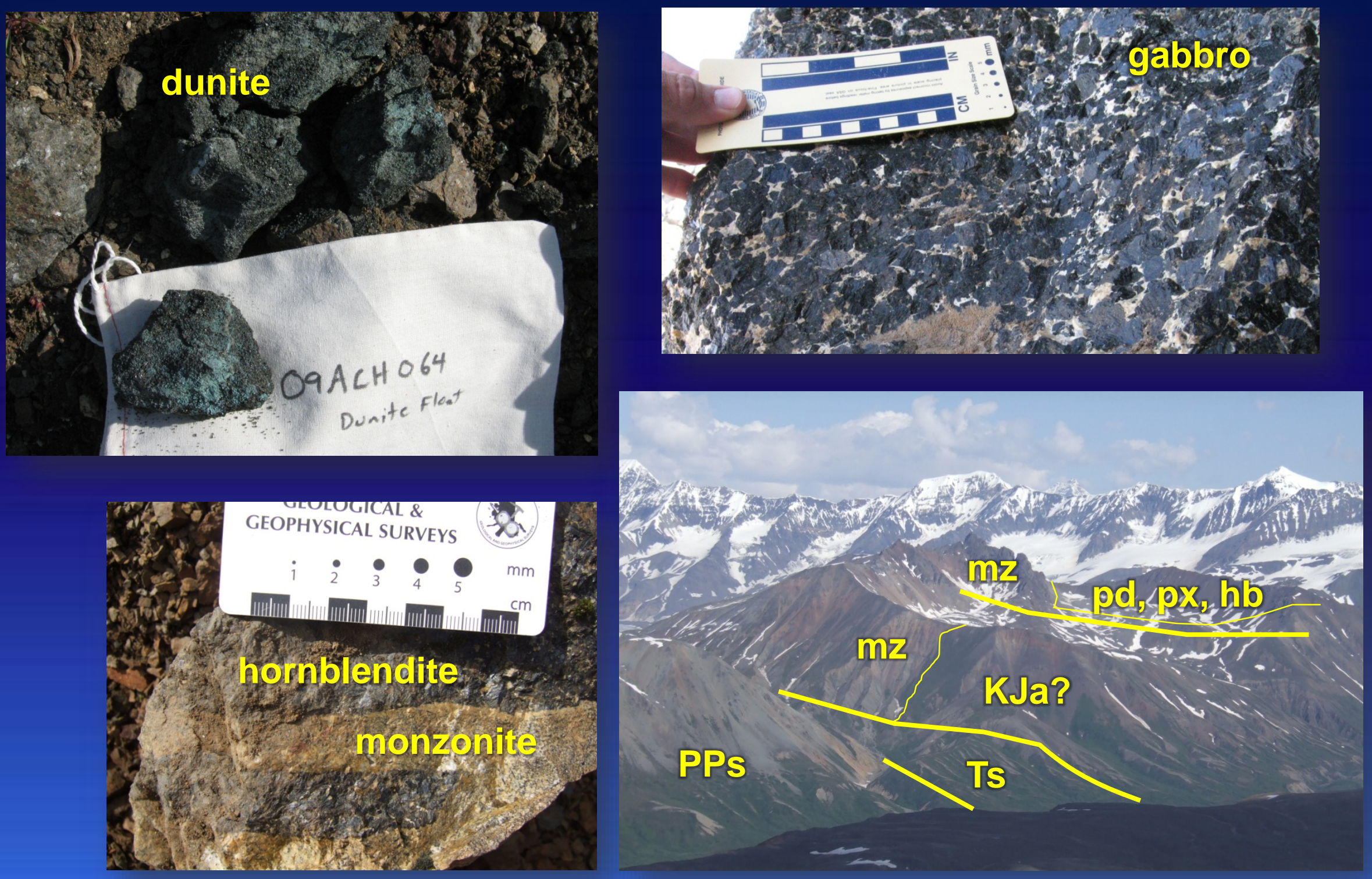


\section{Triassic(?) mafic and ultramafic rocks}

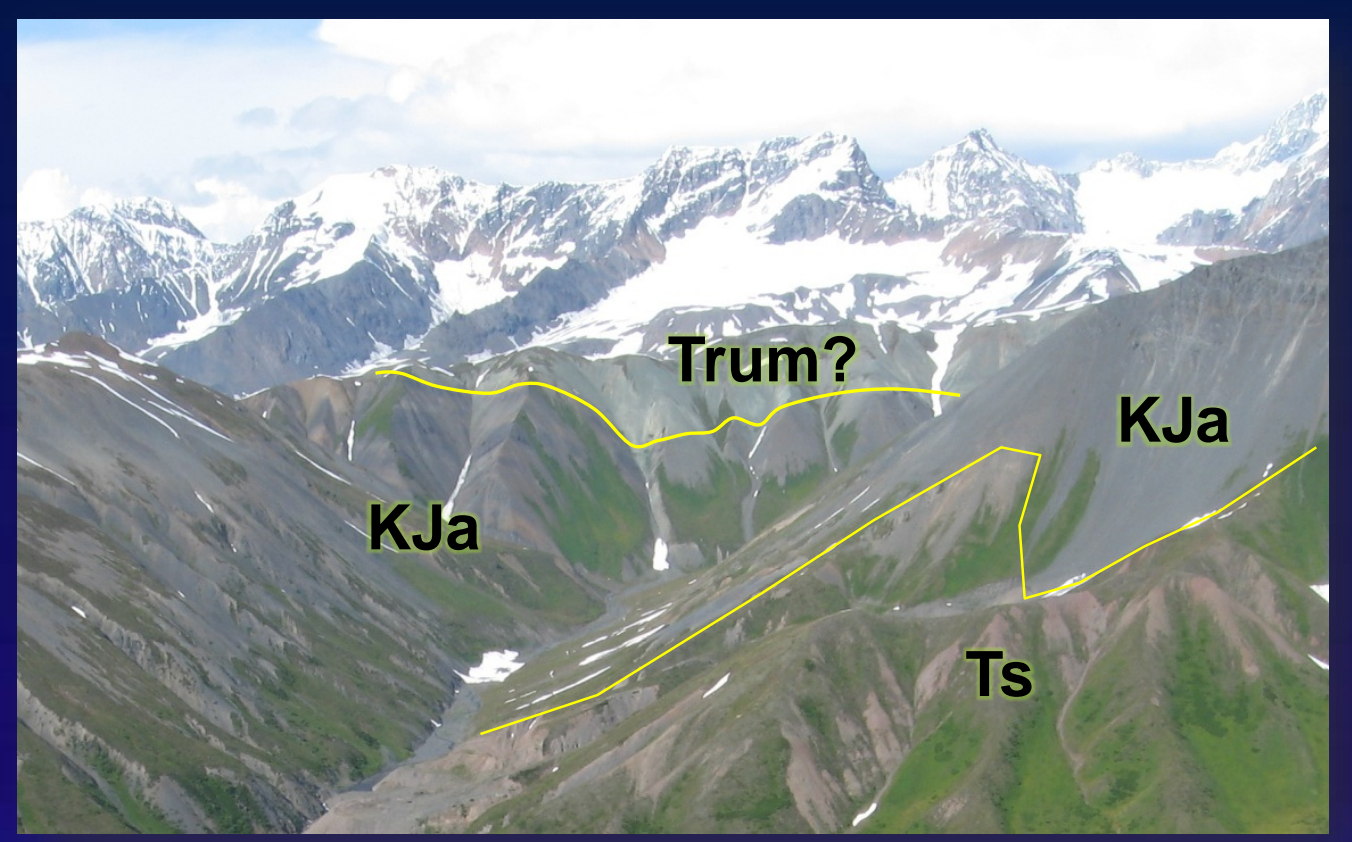

Serpentinized thrust sheet
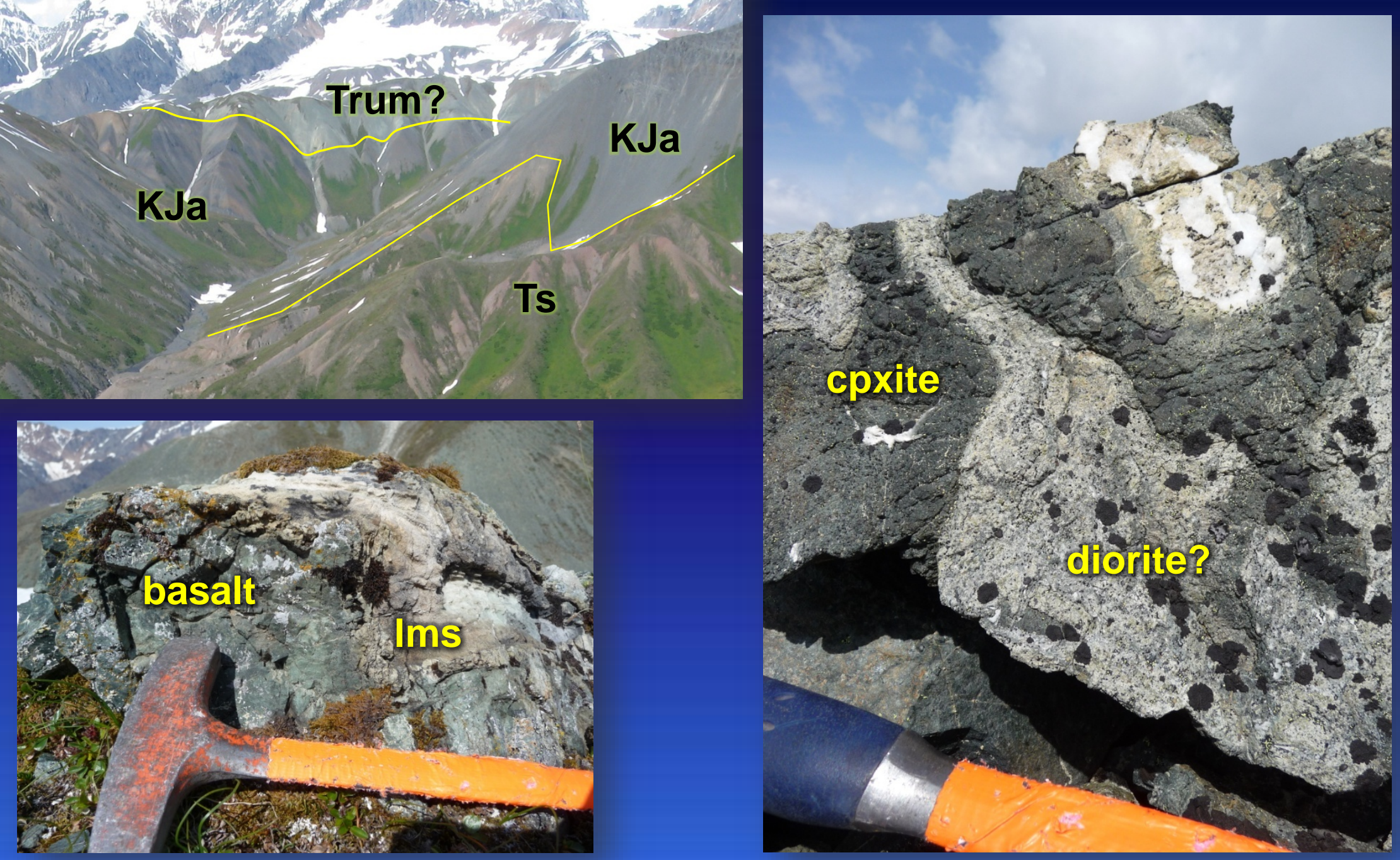


\section{Pending analyses}

- Au, geochem \pm PGE (57)

- Major oxide/TE (120)

- Thin sections (251)

- Slab XRF (260)

- Modal analysis of igneous rocks

- Macrofossils (27)

- Ar-Ar ages ( 6)

- Palynology (3)

- Detrital zircon ages (2)

- Pebble/sand grain counts

Chistochina Glacier 


\section{Pm outta herreio}

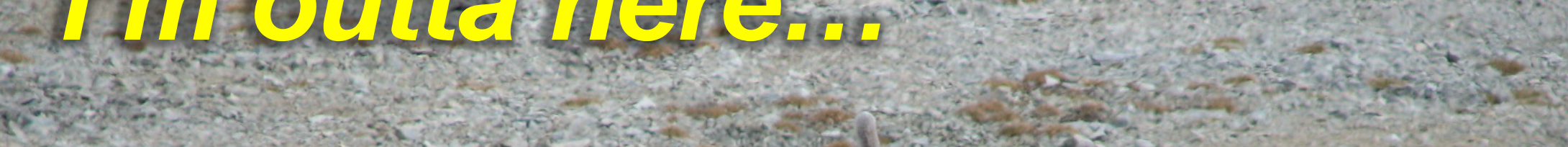

2.

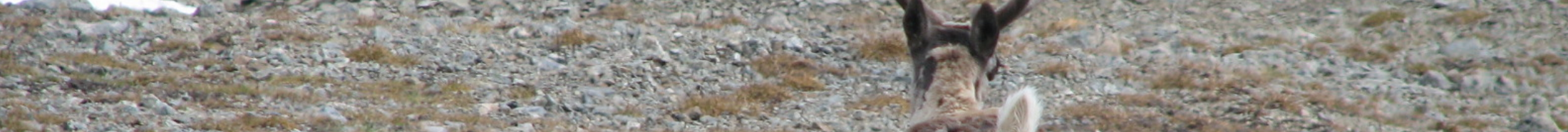

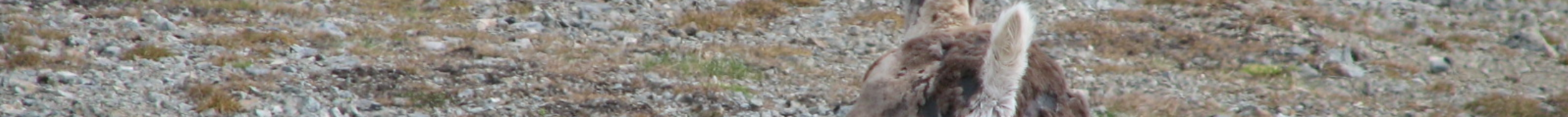

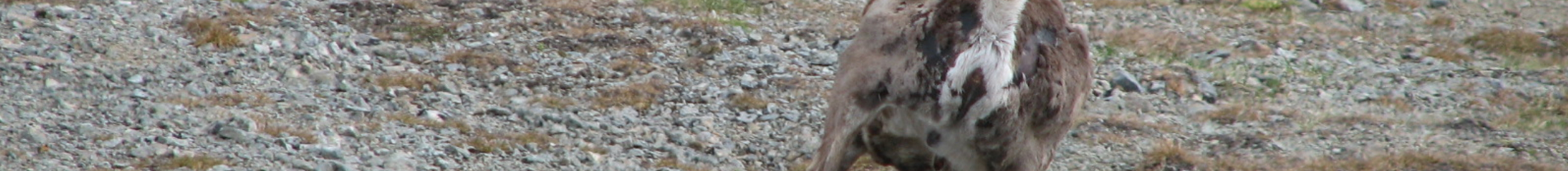

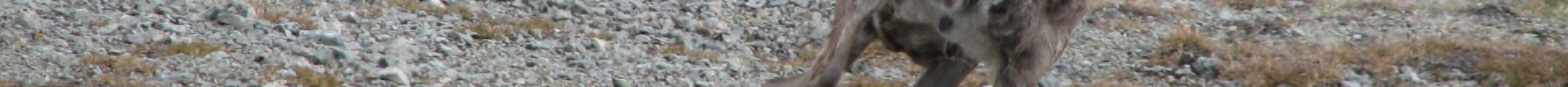

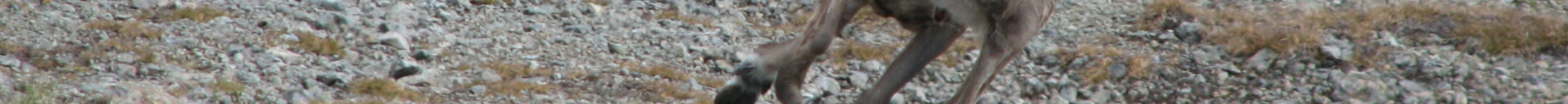

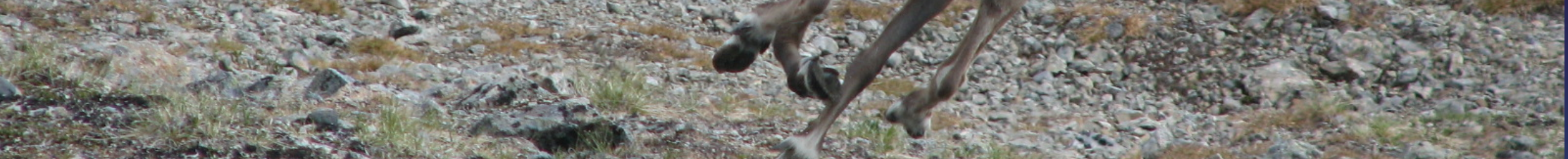

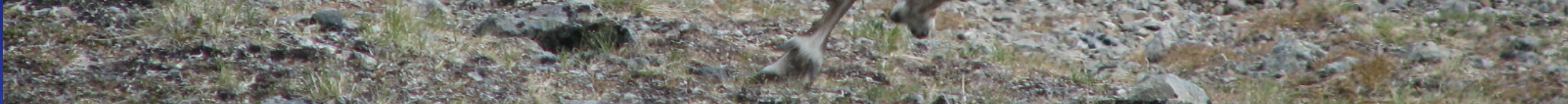

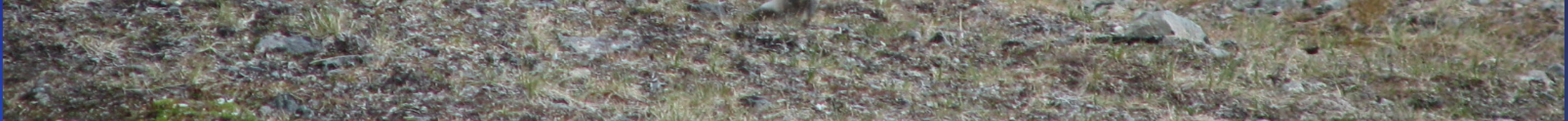

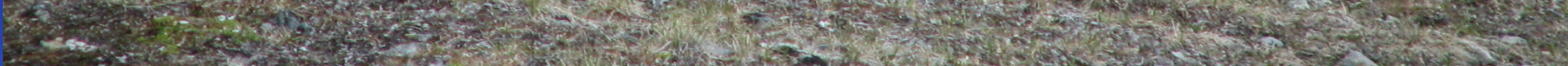

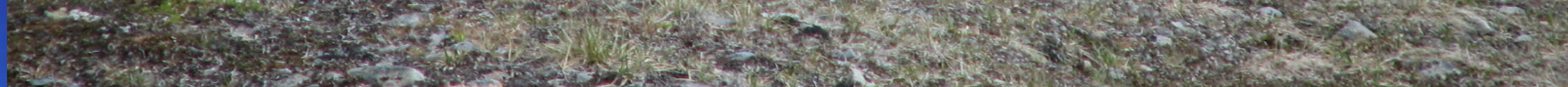

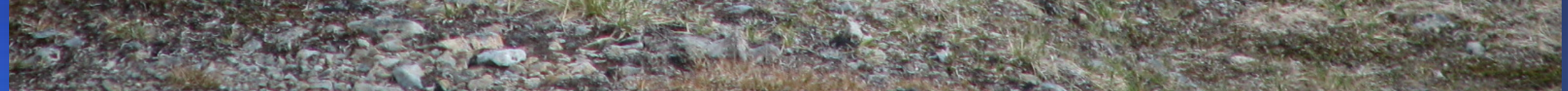

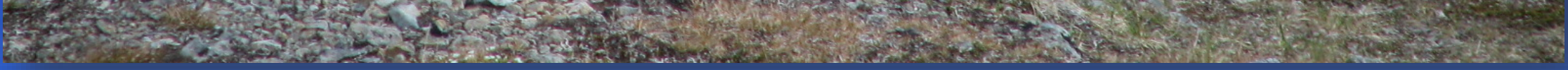

